\title{
Shape transitions in odd-mass $\gamma$-soft nuclei within the interacting boson-fermion model based on the Gogny energy density functional
}

\author{
K. Nomura, ${ }^{1,2}$ R. Rodríguez-Guzmán, ${ }^{3}$ and L. M. Robledo ${ }^{4,5}$ \\ ${ }^{1}$ Physics Department, Faculty of Science, University of Zagreb, HR-10000 Zagreb, Croatia \\ ${ }^{2}$ Center for Computational Sciences, University of Tsukuba, Tsukuba 305-8577, Japan \\ ${ }^{3}$ Physics Department, Kuwait University, 13060 Kuwait, Kuwait \\ ${ }^{4}$ Departamento de Física Teórica, Universidad Autónoma de Madrid, E-28049 Madrid, Spain \\ ${ }^{5}$ Center for Computational Simulation, Universidad Politécnica de Madrid, \\ Campus de Montegancedo, Boadilla del Monte, 28660-Madrid.
}

(Dated: July 30, 2021)

\begin{abstract}
The interacting boson-fermion model (IBFM), with parameters determined from the microscopic Hartree-Fock-Bogoliubov (HFB) approximation, based on the parametrization D1M of the Gogny energy density functional (EDF), is employed to study the structural evolution in odd-mass $\gamma$-soft nuclei. The deformation energy surfaces of even-even nuclei, single-particle energies and occupation probabilities of the corresponding odd-mass systems have been obtained within the constrained HFB approach. Those building blocks are then used as a microscopic input to build the IBFM Hamiltonian. The coupling constants of the boson-fermion interaction terms are taken as free parameters, fitted to reproduce experimental low-lying spectra. The diagonalization of the IBFM Hamiltonian provides the spectroscopic properties for the studied odd-mass nuclei. The procedure has been applied to compute low-energy excitation spectra and electromagnetic transition rates, in the case of the $\gamma$-soft odd-mass systems ${ }^{129-137} \mathrm{Ba},{ }^{127-135} \mathrm{Xe},{ }^{129-137} \mathrm{La}$ and ${ }^{127-135} \mathrm{Cs}$. The calculations provide a reasonable agreement with the available experimental data and agree well with previous results based on the relativistic mean-field approximation.
\end{abstract}

\section{INTRODUCTION}

The microscopic description of odd-mass nuclei is one of the most challenging topics in today's low-energy nuclear structure physics [1]. In those systems, the interplay between the single-particle and collective degrees of freedom plays a key role. Among the theoretical approaches that are nowadays becoming a standard tool for microscopic nuclear structure studies are, the large-scale Shell Model (SM) 22 and self-consistent mean-field (SCMF) methods based on nuclear energy density functionals (EDFs) [3 6]. These theoretical approaches have already been successfully employed in the study of the properties of even-even nuclei. However, a systematic investigation of odd-mass nuclei, particularly in the mediumand heavy-mass regions of the nuclear chart, still represents a major computational challenge.

On the one hand, the SM framework includes the relevant nuclear correlations via the diagonalization of the effective Hamiltonian, defined in a given valence space, and allows a direct access to spectroscopic properties. However, in heavy and/or open-shell nuclei, the dimension of the SM Hamiltonian matrix becomes too large so as to make the approach computationally less feasible. On the other hand, the SCMF methods allow a global description of the intrinsic properties all over the nuclear chart. Nevertheless, in order to describe spectroscopic properties, one has to go beyond the mean-field level to include dynamical correlations arising from the restoration of the (spontaneously) broken symmetries and/or fluctuations in the collective parameters. This can be done within the framework the symmetry-projected generator coordinate method (GCM) 3, 4, 6, 7]. For odd-mass nuclei, however, the GCM framework should be extended to explicitly take into account both the time-odd fields and the effect of blocking at each deformation on the energy surface of a given nuclear system [8. Such a calculation, however, tends to be highly demanding from a computational point of view and indeed has been mainly limited to light nuclei $[8,9$.

The computational difficulties already mentioned, suggest the exploration of alternative schemes that can be used for odd-mass nuclei with arbitrary masses. Examples of such approaches are, the particle-vibration coupling scheme 1, 10 based on both nonrelativistic [11 14 and relativistic [15, 16, EDFs as well as the symmetry-based approaches [17 21. Here, we also mention other fully-microscopic approaches to odd-nucleon systems such as coupled-cluster calculations 22 and the equation of motion phonon method [23, 24]. Such approaches resort to realistic nucleon-nucleon interactions though they have so far been limited to light nuclear systems.

Recently, a method has been developed to describe odd-mass nuclear systems 25], based on the nuclear EDF framework and the particle-core coupling scheme. In this case, the even-even core is described in terms of bosonic degrees of freedom, i.e., the interacting boson model (IBM) [26, and the particle-boson coupling is taken into account within the interacting boson-fermion model (IBFM) 19. The quadrupole deformation energy surfaces for the even-even boson-core nucleus, the singleparticle energies as well as the occupation numbers for the considered odd-mass system are all computed within the SCMF method based on a given nuclear EDF. They are then used as a microscopic input to determine the 
boson-core Hamiltonian and the boson-fermion interaction terms. The coupling constants of the boson-fermion interaction are the only free parameters, which are adjusted to selected spectroscopic data. The method has already been tested for axially-deformed odd-mass Sm and $\mathrm{Eu}[25,27,28$ and $\gamma$-soft odd-mass $\mathrm{Ba}, \mathrm{Xe}, \mathrm{La}$ and Cs nuclei in the $A \approx 130$ mass region [29] using the relativistic DD-PC1 30 and the Gogny EDFs. It has been shown, that the method works equally well regardless of the underlying EDF used as microscopic input.

In this paper, we consider the structural evolution between nearly spherical and $\gamma$-soft shapes in the case of odd-mass $\mathrm{Ba}$, Xe, La and Cs nuclei with $A \approx 130$. As will be shown later on in this paper our results, based on the Gogny-EDF, exhibit a similar level of agreement with the experiment as the ones obtained in Ref. 29] corroborating the validity of the method also for $\gamma$-soft nuclei. Once again, both relativistic and/or non-relativistic EDF produce equivalent results.

Until now, a number of purely phenomenological IBFM calculations have already been performed in this mass region 31-37. A virtue of the present approach is that, though its applicability is currently limited to nuclei where spectroscopic data are available, the bosoncore Hamiltonian, single-particle energies and occupation probabilities of the considered odd-mass systems are completely determined by fully microscopic SCMF calculations. We compare our results with those obtained in the phenomenological IBFM studies already mentioned.

The paper is organized as follows. In Sec. [II we briefly outline the method employed and discuss the parameters of the IBFM Hamiltonian. The results for the even-even boson core nuclei ${ }^{128-136} \mathrm{Ba}$ and ${ }^{126-134} \mathrm{Xe}$ are presented in Sec. III In particular, we discuss the corresponding mean-field and (mapped) IBM energy surfaces as well as the low-energy excitation spectra. The spectroscopic properties of the odd-mass nuclei ${ }^{129-137} \mathrm{Ba},{ }^{127-135} \mathrm{Xe}$, ${ }^{129-137} \mathrm{La}$ and ${ }^{127-135} \mathrm{Cs}$ are presented in Sec. IV] where, the computed low-energy positive- and negative-parity excitation spectra, $B(E 2)$ transition strengths, spectroscopic quadrupole and magnetic moments are discussed and compared with the available experimental data. We end up this section with a detailed analysis of the excitation spectrum and electromagnetic properties for a few selected nuclei for which experimental data are available. Finally, Sec. $\mathrm{V}$ is devoted to the conclusions and work perspectives.

\section{DESCRIPTION OF THE MODEL}

In this section, we briefly outline the theoretical framework used in this study [25]. We also discuss the parameters of the IBFM Hamiltonian employed in the calculations.

The IBFM Hamiltonian, used to describe the studied odd-mass nuclei, consists of three terms, i.e, the even-even boson core or Interacting Boson Model (IBM)
Hamiltonian $\hat{H}_{B}$, the single-particle Hamiltonian for unpaired fermions $\hat{H}_{F}$ and the boson-fermion coupling term $\hat{H}_{B F}$

$$
\hat{H}=\hat{H}_{B}+\hat{H}_{F}+\hat{H}_{B F} .
$$

The building blocks of the IBM are the $s$ and $d$ bosons, which represent the collective pairs of valence nucleons 38. coupled to angular momentum $J^{\pi}=0^{+}$and $2^{+}$, respectively. The number of bosons $N_{B}$ and fermions $N_{F}$ are assumed to be conserved separately. We restrict ourselves to the simplest case $N_{F}=1$, where contributions from three or higher quasiparticle configurations are not included. In addition, no distinction is made between neutron and proton bosons.

The IBM Hamiltonian $\hat{H}_{B}$ reads

$$
\hat{H}_{B}=\epsilon_{d} \hat{n}_{d}+\kappa \hat{Q}_{B} \cdot \hat{Q}_{B},
$$

and is given in terms of the $d$-boson number operator $\hat{n}_{d}=d^{\dagger} \cdot \tilde{d}$, and the quadrupole operator $\hat{Q}_{B}=s^{\dagger} \tilde{d}+$ $d^{\dagger} \tilde{s}+\chi\left[d^{\dagger} \times \tilde{d}\right]^{(2)}$. The quantities $\epsilon_{d}, \kappa$, and $\chi$ represent parameters of the Hamiltonian $\hat{H}_{B}$.

On the other hand, the single-fermion Hamiltonian takes the form

$$
\hat{H}_{F}=\sum_{j} \epsilon_{j}\left[a_{j}^{\dagger} \times \tilde{a}_{j}\right]^{(0)}
$$

where $a_{j}^{\dagger}$ is the fermion creation operator for the orbital $j$ and $\epsilon_{j}$ stands for the corresponding single-particle energy.

For the boson-fermion coupling Hamiltonian $\hat{H}_{B F}$ we have employed the simplest possible form that has been shown to be most relevant in the phenomenological studies of Ref [19:

$$
\begin{aligned}
\hat{H}_{B F}= & \sum_{j j^{\prime}} \Gamma_{j j^{\prime}} \hat{Q}_{B} \cdot\left[a_{j}^{\dagger} \times \tilde{a}_{j^{\prime}}\right]^{(2)} \\
& +\sum_{j j^{\prime} j^{\prime \prime}} \Lambda_{j j^{\prime}}^{j^{\prime \prime}}:\left[\left[d^{\dagger} \times \tilde{a}_{j}\right]^{\left(j^{\prime \prime}\right)} \times\left[a_{j^{\prime}}^{\dagger} \times \tilde{d}\right]^{\left(j^{\prime \prime}\right)}\right]^{(0)}: \\
& +\sum_{j} A_{j}\left[a^{\dagger} \times \tilde{a}_{j}\right]^{(0)} \hat{n}_{d} .
\end{aligned}
$$

The first, second and third terms are referred to as the dynamical quadrupole, exchange, and monopole interactions, respectively. For the strength parameters $\Gamma_{j j^{\prime}}, \Lambda_{j j^{\prime}}^{j^{\prime \prime}}$ and $A_{j}$ we have used the following expressions which were derived within the generalized seniority scheme [18:

$$
\begin{aligned}
\Gamma_{j j^{\prime}} & =\Gamma_{0} \gamma_{j j^{\prime}} \\
\Lambda_{j j^{\prime}}^{j^{\prime \prime}} & =-2 \Lambda_{0} \sqrt{\frac{5}{2 j^{\prime \prime}+1}} \beta_{j j^{\prime \prime}} \beta_{j^{\prime} j^{\prime \prime}} \\
A_{j} & =-A_{0} \sqrt{2 j+1}
\end{aligned}
$$

The quantities $\gamma_{j j^{\prime}}=\left(u_{j} u_{j^{\prime}}-v_{j} v_{j^{\prime}}\right) Q_{j j^{\prime}}$ and $\beta_{j j^{\prime}}=$ $\left(u_{j} v_{j^{\prime}}+v_{j} u_{j^{\prime}}\right) Q_{j j^{\prime}}$, are given in terms of the occupation probabilities $u_{j}$ and $v_{j}$ for the orbital $j$ (satisfying $u_{j}^{2}+v_{j}^{2}=1$ ) and the matrix element of the quadrupole 
operator in the single-particle basis $Q_{j j^{\prime}}=\left\langle j\left\|Y^{(2)}\right\| j^{\prime}\right\rangle$. Furthermore, $\Gamma_{0}, \Lambda_{0}$ and $A_{0}$ denote strength parameters. For a detailed account of the formulas in Eqs. (4)-(7), as well as a discussion of relevant applications to odd-mass nuclei, the reader is referred to Ref. [18].

The first step to build the full IBFM Hamiltonian, $\hat{H}$ Eq. (1), is to fix the parameters of the IBM Hamiltonian $\hat{H}_{B}$ by using the fermion-to-boson mapping procedure developed in Refs. [39, 40]. Within this context, the fermionic $(\beta, \gamma)$-deformation energy surface, obtained via mean-field calculations based on the parametrization D1M [41] of the Gogny-EDF, is mapped onto the expectation value of $\hat{H}_{B}$ in the boson condensate state as defined in Ref [42. This procedure completely determines the parameters $\epsilon_{d}, \kappa$ and $\chi$. Calculations have also been carried out with the parametrization D1S [43] of the Gogny-EDF. However, as the results turn out to be rather similar to the ones provided by the Gogny-D1M EDF, we will just discuss the latter in this work.

For a more detailed account of the constrained GognyHFB approximation the reader is referred to Refs. 44, 45. Details of the fermion-to-boson mapping procedure for even-even system can be found in Refs. [39, 40]. The parameters derived for the considered boson-core nuclei ${ }^{128-136} \mathrm{Ba}$ and ${ }^{126-134} \mathrm{Xe}$ are listed in Table I.

TABLE I. Parameters of the boson Hamiltonian $\hat{H}_{B}$ for ${ }^{128-136} \mathrm{Ba}$ and ${ }^{126-134} \mathrm{Xe}$. The values of $\epsilon_{d}$ and $\kappa$ are in $\mathrm{MeV}$, while $\chi$ is dimensionless.

\begin{tabular}{llll}
\hline \hline & $\epsilon_{d}$ & $\kappa$ & $\chi$ \\
${ }^{128} \mathrm{Ba}$ & 0.120 & -0.080 & -0.14 \\
${ }^{130} \mathrm{Ba}$ & 0.150 & -0.081 & -0.16 \\
${ }^{132} \mathrm{Ba}$ & 0.265 & -0.081 & -0.10 \\
${ }^{134} \mathrm{Ba}$ & 0.620 & -0.083 & -0.37 \\
${ }^{136} \mathrm{Ba}$ & 1.000 & -0.090 & -0.80 \\
${ }^{126} \mathrm{Xe}$ & 0.245 & -0.079 & -0.13 \\
${ }^{128} \mathrm{Xe}$ & 0.280 & -0.079 & -0.22 \\
${ }^{130} \mathrm{Xe}$ & 0.375 & -0.081 & -0.12 \\
${ }^{132} \mathrm{Xe}$ & 0.620 & -0.086 & -0.30 \\
${ }^{134} \mathrm{Xe}$ & 1.000 & -0.090 & -0.87 \\
\hline \hline
\end{tabular}

For all the nuclei considered in this work ${ }^{129-137} \mathrm{Ba}$, ${ }^{127-135} \mathrm{Xe},{ }^{129-137} \mathrm{La}$ and ${ }^{127-135} \mathrm{Cs}$ we have considered as the fermion valence space (neutrons for $\mathrm{Ba}$ and $\mathrm{Xe}$ and protons for $\mathrm{La}$ and $\mathrm{Cs}$ ), all the spherical singleparticle orbitals between magic numbers 50 and 82, i.e., $3 s_{1 / 2}, 2 d_{3 / 2}, 2 d_{5 / 2}$ and $1 g_{7 / 2}$ for positive-parity states and $1 h_{11 / 2}$ for negative-parity states. The single-particle energies $\epsilon_{j}$ and the occupation probabilities $v_{j}^{2}$ are obtained from Gogny-D1M HFB calculations at the spherical configuration [25. In those calculations, for a given odd-mass nucleus with the odd neutron (proton) number $N_{0}\left(Z_{0}\right)$, the standard even number parity constrained Gogny-HFB approach (i.e., without blocking) has been employed but using $N_{0}\left(Z_{0}\right)$ for the neutron (proton) number constraint. The single-particle energies and oc- cupation probabilities obtained for the considered odd- $A$ nuclei, are shown in Figs. 1 and 2 , respectively.

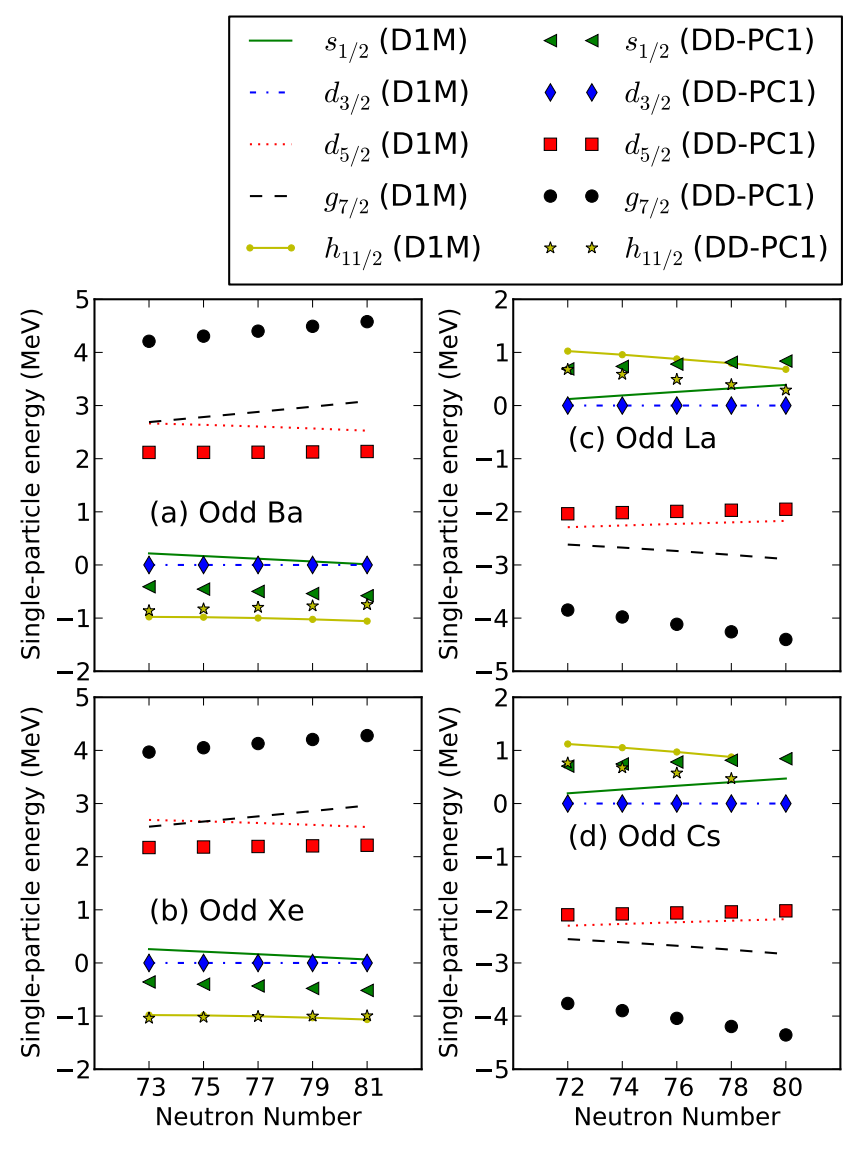

FIG. 1. (Color online) The single-particle energies corresponding to the $3 s_{1 / 2}, 2 d_{3 / 2}, 2 d_{5 / 2}, 1 g_{7 / 2}$ and $1 h_{11 / 2}$ orbitals employed in the present study (denoted as "D1M") for the considered odd-mass nuclei are plotted with respect to the $2 d_{3 / 2}$ single-particle level. Results from Ref. 29] are also included in the plot (denoted as "DD-PC1").

The coupling constants of the boson-fermion interaction term $\hat{H}_{B F}\left(\Gamma_{0}, \Lambda_{0}\right.$ and $\left.A_{0}\right)$ are treated as free parameters. They are fitted, for each nucleus and separately for positive- and negative-parity states, to a few experimental excitation spectra 25. The single-particle energies calculated within the Gogny-HFB method are somewhat at variance with those employed in purely phenomenological IBFM studies in this mass region 33, 34, 36. For instance, in Ref. 36] for the odd- $A$ Ba and Xe nuclei, the $3 s_{1 / 2}$ orbital lies around $0.4 \mathrm{MeV}$ below the $2 d_{3 / 2}$ orbital. In the present study, on the other hand, the two orbitals are nearly degenerated as can be seen from Figs.1(a) and 1(b). In order to better reproduce the ordering of the energy levels, we have assumed that the monopole strength parameter for the positive-parity states, denoted by $A_{0}^{+}$, is allowed to be $j$ dependent, i.e., $A_{0}^{+} \equiv A_{j}^{\prime}$. A similar assumption was made in the previous study [29].

The fitted strength parameters of the boson-fermion 


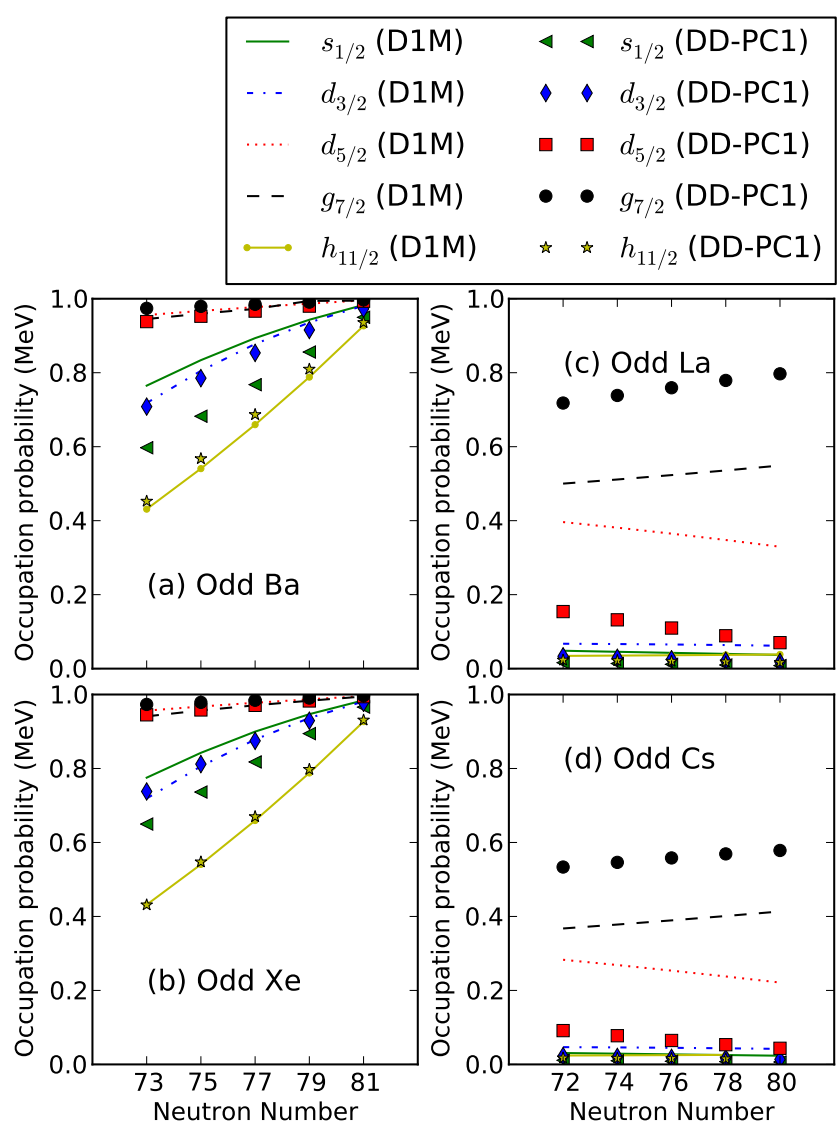

FIG. 2. (Color online) The same as in Fig. 1 but for occupation probabilities of the $3 s_{1 / 2}, 2 d_{3 / 2}, 2 d_{5 / 2}, 1 g_{7 / 2}$ and $1 h_{11 / 2}$ orbitals.

interaction terms $\Gamma_{0}^{ \pm}$and $\Lambda_{0}^{ \pm}$are plotted in Figs. 3 and 4 . respectively. On the other hand, the monopole strength parameters $A_{j}^{\prime}$ are listed in Table II The behavior of some of the fitted parameters, as functions of the nucleon number, reflects structural changes along a given isotopic chain. For instance, one observes kinks in the $\Gamma_{0}^{+}$values for the odd- $A$ La and Cs around the neutron number $N=78$ (Figs. 3(c) and 3(d)) and in the $\Lambda_{0}^{+}$ones for the odd- $A$ Ba and Xe around $N=79$ (Fig. 4(a) and 4(b)). As will be shown, this is supported by the deformation energy surfaces for the boson-core nuclei with those neutron numbers exhibiting flat-bottomed potential characteristic of transitional systems (see, Figs. 5 and 6).

Here, we briefly summarize some differences and similarities between the IBFM parameters employed in the present study and those obtained in Ref. 29]:

- Major differences are found in the parameters $\epsilon_{d}$ and $\kappa$ of the IBM Hamiltonian obtained in this work and the ones of Ref. 29]. In particular, the value of $\epsilon_{d}(\kappa)$ used in Ref. 29] is significantly smaller (larger) than the one obtained in this work. To a large extent, these differences reflect the ones

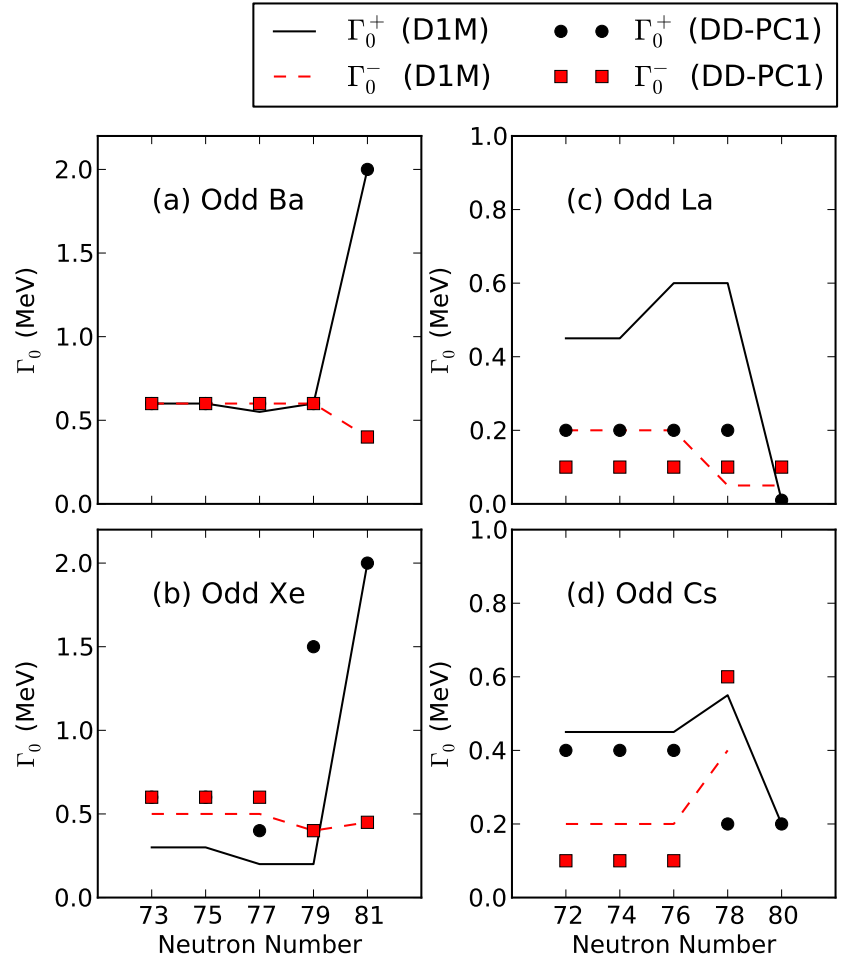

FIG. 3. (Color online) The strength parameter $\Gamma_{0}$ Eq. (5) (denoted by "D1M") is plotted for both positive- $\left(\Gamma_{0}^{+}\right)$and negative-parity $\left(\Gamma_{0}^{-}\right)$states. Results from Ref. 29] are also included in the plot (denoted as "DD-PC1").

between the Gogny-D1M and DD-PC1 energy surfaces.

- From the comparison of the single-particle energies in Figs. 1(a) and 1(b), one realizes that the ordering of the $3 s_{1 / 2}$ and $2 d_{3 / 2}$ orbitals is different for the Gogny-D1M and the relativistic DD-PC1 EDFs. In addition, all five Gogny-D1M single-particle levels lie closer to each other than those provided by the DD-PC1 EDF [29].

- As seen from Fig. 2(a) and 2(b), the occupation probabilities $v_{j}^{2}$ for the odd- $A \mathrm{Ba}$ and Xe nuclei are similar for both Gogny-D1M and DD-PC1 results, except for the $v_{s_{1 / 2}}^{2}$ values. On the other hand, for the odd- $A$ La (Fig. 2(c)) and Cs (Fig. 2(d)) nuclei, the values of $v_{g_{7 / 2}}^{2}$ and $v_{d_{5 / 2}}^{2}$ are substantially different from those obtained with the DD-PC1 EDF.

- As can be seen from Figs. 3 and 4, similar values are obtained for the parameters $\Gamma_{0}^{-}$and $\Lambda_{0}^{-}$ as compared to the ones in Ref. 29]. There are, however, considerable differences in the $\Gamma_{0}^{+}$and $\Lambda_{0}^{+}$ values. This reflects the quantitative differences in the $\epsilon_{j}$ and $v_{j}^{2}$ values provided by the Gogny-D1M and DD-PC1 approaches. On the other hand, the $A_{j}^{\prime}$ values are generally smaller in magnitude than 

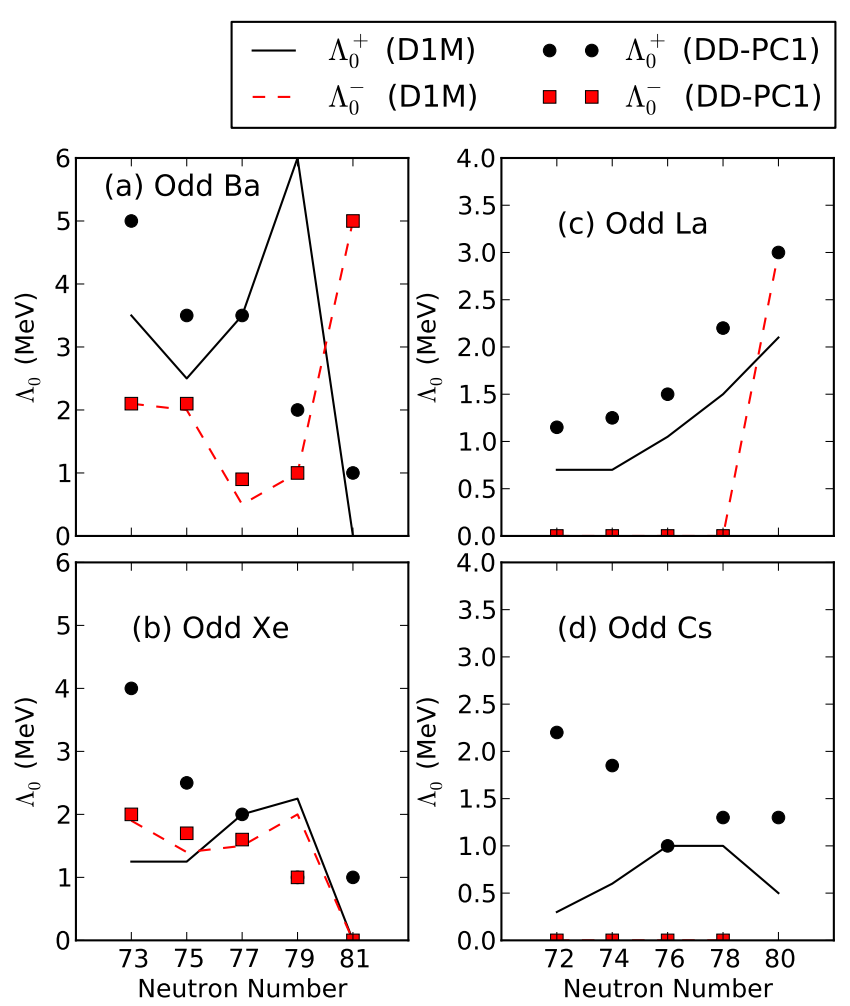

FIG. 4. (Color online) The same as in Fig. 3 , but for the strength parameter $\Lambda_{0}$ of the exchange term Eq. (6).

TABLE II. The strength parameters of the monopole terms $A_{j}^{\prime}$. All entries are in $\mathrm{MeV}$.

\begin{tabular}{llllll}
\hline \hline & $A_{1 / 2}^{\prime}$ & $A_{3 / 2}^{\prime}$ & $A_{5 / 2}^{\prime}$ & $A_{7 / 2}^{\prime}$ & $A_{11 / 2}^{\prime}$ \\
${ }^{129} \mathrm{Ba}$ & -0.23 & & & -0.58 & \\
${ }^{131} \mathrm{Ba}$ & & & & & \\
${ }^{133} \mathrm{Ba}$ & & & & & -0.5 \\
${ }^{135} \mathrm{Ba}$ & & -0.35 & & & -0.3 \\
${ }^{137} \mathrm{Ba}$ & & -1.2 & & -0.64 & \\
${ }^{127} \mathrm{Xe}$ & -0.22 & & & & \\
${ }^{129} \mathrm{Xe}$ & -0.10 & & & -0.15 \\
${ }^{131} \mathrm{Xe}$ & & & & -0.15 \\
${ }^{133} \mathrm{Xe}$ & & -0.25 & & -0.40 \\
${ }^{135} \mathrm{Xe}$ & & -1.5 & & & \\
${ }^{129} \mathrm{La}$ & & & -0.35 & & \\
${ }^{131} \mathrm{La}$ & & & -0.40 & & -0.10 \\
${ }^{133} \mathrm{La}$ & & & -0.22 & & \\
${ }^{135} \mathrm{La}$ & & & -0.25 & & \\
${ }^{137} \mathrm{La}$ & & & & & \\
${ }^{127} \mathrm{Cs}$ & & & -0.05 & & \\
${ }^{129} \mathrm{Cs}$ & & & -0.94 & & \\
${ }^{131} \mathrm{Cs}$ & & & -0.25 & & \\
${ }^{133} \mathrm{Cs}$ & & & -0.30 & & \\
${ }^{135} \mathrm{Cs}$ & & & -0.90 & & \\
\hline \hline
\end{tabular}

those in Ref. [29] (see Table II] and Tables IV and $\mathrm{V}$ in Ref. 29]).

Once all the building blocks of the IBFM Hamiltonian $\hat{H}$ Eq. 11 are determined, this Hamiltonian is diagonalized in the spherical basis $|j, L, \alpha, J\rangle$ [46], where $L$ and $J$ stand for the angular momenta of the boson and bosonfermion systems, respectively. On the other hand, $\alpha$ represents a set of $\mathrm{U}(5)$ quantum numbers [26]. Note, that the selection rule $|L-j| \leq J \leq L+j$ must be satisfied. The wave functions resulting from the diagonalization of $\hat{H}$ are then used to compute electromagnetic properties, such as electric quadrupole (E2) and magnetic dipole (M1) transitions.

The E2 transition operator reads

$$
\hat{T}^{(E 2)}=\hat{e}_{B} \hat{Q}_{B}-e_{F} \sum_{j j^{\prime}} \frac{1}{\sqrt{5}} \gamma_{j j^{\prime}}\left[a^{\dagger} \times \tilde{a}_{j^{\prime}}\right]^{(2)},
$$

where the first and second terms represent the boson and fermion E2 operators, respectively. In Eq. (8), $\hat{Q}_{B}$ is the quadrupole operator already defined in Eq. (2) with the same value of the parameter $\chi$. The effective bosonic charge $e_{B}$ is fitted, for each nucleus, to reproduce the experimental $B\left(E 2 ; 2_{1}^{+} \rightarrow 0_{1}^{+}\right)$value of the corresponding even-even boson-core. On the other hand, the effective fermionic charge $e_{F}$ is taken separately for proton $(\pi)$ and neutron $(\nu)$ as $e_{F}^{\pi}=0.25 \mathrm{eb}$ and $e_{F}^{\nu}=0.125 \mathrm{eb}[29$.

The M1 transition operator is given by

$$
\hat{T}^{(M 1)}=\sqrt{\frac{3}{4 \pi}}\left(\hat{T}_{B}^{(M 1)}+\hat{T}_{F}^{(M 1)}\right) .
$$

where $\hat{T}_{B}^{(M 1)}=g_{B} \hat{L}$, with $\hat{L}=\sqrt{10}\left[d^{\dagger} \times \tilde{d}\right]^{(1)}$ being the boson angular momentum operator and the gyromagnetic factor $g_{B}=\mu_{2_{1}^{+}} / 2$ given in terms of the magnetic moment $\mu_{2_{1}^{+}}$of the $2_{1}^{+}$state of the even-even nucleus. The corresponding experimental value has been used for $\mu_{2_{1}^{+}}$. The fermionic part $\hat{T}_{F}^{(M 1)}$ takes the form 18

$$
\hat{T}_{F}^{(M 1)}=-\sum_{j j^{\prime}} g_{j j^{\prime}} \sqrt{\frac{j(j+1)(2 j+1)}{3}}\left[a_{j}^{\dagger} \times \tilde{a}_{j^{\prime}}\right]^{(1)},
$$

with the coefficients $g_{j j^{\prime}}$ given by

$g_{j j^{\prime}}= \begin{cases}\frac{(2 j-1) g_{l}+g_{s}}{2 j} & \left(j=j^{\prime}=l+\frac{1}{2}\right) \\ \frac{(2 j+3) g_{l}-g_{s}}{2(j+1)} & \left(j=j^{\prime}=l-\frac{1}{2}\right) \\ \left(g_{l}-g_{s}\right) \sqrt{\frac{2 l(l+1)}{j(j+1)(2 j+1)(2 l+1)}} & \left(j^{\prime}=j-1 ; l=l^{\prime}\right)\end{cases}$

where $l$ represents the orbital angular momentum of the single-particle state. The fermion $g_{l}$ and $g_{s}$ gyromagnetic factors take the usual Schmidt values $g_{l}=1.0$ $\mu_{N}^{2}$ and $g_{s}=5.58 \mu_{N}^{2}$ for the proton and $g_{l}=0$ and $g_{s}=-3.82 \mu_{N}^{2}$ for the neutron. The $g_{s}$ value has been quenched by $30 \%$ for both the protons and neutrons [25. 47. 


\section{RESULTS FOR THE EVEN-EVEN CORE NUCLEI}

In this section, we show that the IBM Hamiltonian, with the parameters determined by mapping the GognyD1M energy surface onto the expectation value of that Hamiltonian, provides a reasonable description of the considered even-even core nuclei.

The $(\beta, \gamma)$-deformation energy surfaces obtained for the nuclei ${ }^{128-136} \mathrm{Ba}$ and ${ }^{126-134} \mathrm{Xe}$ within the constrained Gogny-D1M HFB method are shown in Fig. 5. They suggest structural evolution from notably $\gamma$-soft $\left({ }^{128,130} \mathrm{Ba}\right.$ and $\left.{ }^{126,128} \mathrm{Xe}\right)$ to nearly spherical $\left({ }^{136} \mathrm{Ba}\right.$ and ${ }^{134} \mathrm{Xe}$ ) shapes. There are also transitional regions around ${ }^{132,134} \mathrm{Ba}$ and ${ }^{130,132} \mathrm{Xe}$. In many cases, one observes a shallow triaxial minimum with $\gamma=0^{\circ}-20^{\circ}$. In general, the Gogny-D1M surfaces for the Xe nuclei look softer along the $\gamma$-direction than those for the Ba neighbors.

Of particular interest is ${ }^{134} \mathrm{Ba}$, identified as the first empirical evidence [49] of the E(5) critical-point symmetry [50] of the second-order quantum phase transition between spherical vibrational $\mathrm{U}(5)$ and $\gamma$-soft $\mathrm{O}(6)$ dynamical symmetries. The $\mathrm{E}(5)$ model is derived from the five-dimensional collective Hamiltonian using a collective potential with an infinite square-well along $\beta$ and independent of the $\gamma$ deformation 50. As can be seen from Fig. 5, the Gogny-D1M energy surface for ${ }^{134} \mathrm{Ba}$ looks almost flat for $0 \leq \beta \leq 0.15$. It is also flat along the $\gamma$ direction. In fact, among all the studied even-even $\mathrm{Ba}$ and Xe nuclei, it is the one that best resembles the $\mathrm{E}(5)$ potential. Note, that the Gogny-D1M energy surfaces depicted in Fig. 5 are, rather similar to those obtained within the relativistic framework based on the DD-PC1 30. EDF (see, Ref. 29]).

The mapped IBM surfaces are displayed in Fig. 6 . They exhibit a similar systematic trend, as functions of the neutron number, as the original Gogny-D1M energy surfaces in Fig. 5. Despite the fact that only three parameters $\left(\epsilon_{d}, \kappa\right.$ and $\left.\chi\right)$ have been introduced to determine the IBM Hamiltonian, the mapped surfaces reproduce the most relevant feature of the Gogny-D1M ones: $\gamma$-softness in the vicinity of the minimum. On the other hand, several discrepancies are also observed. At variance with the mean-field ones, the IBM surfaces are generally flat far from the minimum, i.e., $\beta \geq 0.2$. One of the reasons for the discrepancy could be that we have paid much attention to reproducing the topology of the Gogny-D1M energy surface in the neighborhood of the minimum as we expect that configurations around the minimum would be the most relevant for the low-energy collective dynamics. Another reason is that, like in previous studies, the present IBM framework only comprises limited types (i.e., $J^{\pi}=0^{+}$and $2^{+}$) as well as number of valence nucleon pairs. Furthermore, it employs the simplified form of the Hamiltonian Eq. 22, whereas the HFB framework contains more degrees of freedom and, as a consequence, provides energy surfaces far richer in topology than the IBM ones. Moreover, in the case of lighter $\mathrm{Ba}$ and Xe isotopes, the IBM surfaces do not reproduce the shallow triaxial minimum that is found in the Gogny-D1M ones, but exhibit a minimum only at $\gamma=0^{\circ}$. This is because, as seen from the analytical expression of the energy surface [42, any two-body IBM-1 Hamiltonian never gives rise to a triaxial minimum. To produce such a minimum, it is necessary to include a three-body boson term [51. Such a term is also important to better describe $\gamma$-band levels, while it makes a marginal contribution to the ground-state band. For this reason, and for the sake of simplicity, we have not taken it into account in the considered IBM Hamiltonian.

Even though the deformation energy surfaces provide useful information about shape phase transitions at the mean-field level, a more quantitative analysis requires the computation of spectroscopic properties. To this end, in Fig. 7 we have plotted the low-energy spectra obtained for the considered even-even $\mathrm{Ba}$ and Xe nuclei as functions of the neutron number. They are compared with the available experimental data [48. Our results for the low-lying excitation spectra (panels (a) and (c) of Fig. 7) exhibit, a reasonable agreement with the experimental ones (see, panels (b) and (d)), exception made of the overestimation of the $6_{1}^{+}$level at $N=80$. The spectra predicted for even-even Ba nuclei exhibit a steady increase with neutron number (see, Fig. 7(a)). The near doublet $\left(4_{1}^{+}, 2_{2}^{+}\right)$, which is seen for $72 \leq N \leq 78$, suggests $\gamma$-softness. At $N=80$, one notices the $\left(4_{1}^{+}, 2_{2}^{+}, 0_{2}^{+}\right)$ triplet, characteristic of a vibrational nucleus. As seen from Fig. 7(c), a similar overall trend has been obtained for even-even Xe nuclei. Though the spectra obtained in Ref. 29] are similar, they are more stretched than those obtained in the present work.

\section{RESULTS FOR THE ODD- $A$ NUCLEI}

In this section, we turn our attention to the odd-mass nuclei ${ }^{129-137} \mathrm{Ba},{ }^{127-135} \mathrm{Xe},{ }^{129-137} \mathrm{La}$ and ${ }^{127-135} \mathrm{Cs}$. We discuss the overall trend of the computed excitation spectra (Sec. IV A) as well as electromagnetic transitions (Sec. IV B in each isotopic chain as functions of the nucleon number. We then present more detailed spectroscopic results for some selected nuclei (Sec. IV C).

\section{A. Systematics of low-energy spectra}

In Figs. 8 to 11 , the low-energy positive- and negativeparity excitation spectra obtained for the considered oddmass $\mathrm{Ba}, \mathrm{Xe}, \mathrm{La}$ and $\mathrm{Cs}$ nuclei are depicted as functions of the neutron number. They have been obtained via the diagonalization of the IBFM Hamiltonian with the parameters shown in Tables I and II (see also, Figs. 1 4 ). For each isotopic chain, the predicted spectra are compared with the available experimental values in Ref. [48. Those spectra are in reasonable good agreement with the experiment. 

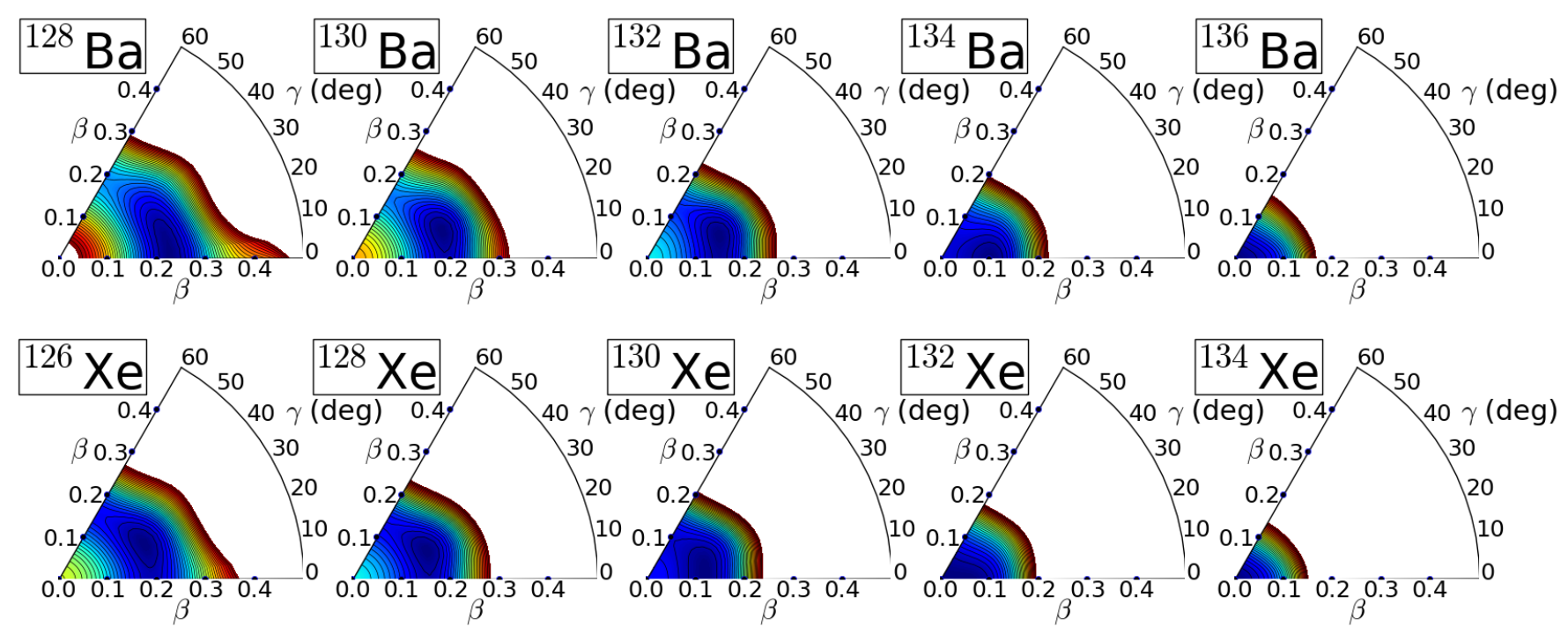

FIG. 5. (Color online) The Gogny-D1M energy surfaces for the even-even nuclei ${ }^{128-136}$ Ba (upper panel) and ${ }^{126-134}$ Xe (lower panel) are plotted up to $3 \mathrm{MeV}$ above the absolute minimum. The difference between neighboring contours is $100 \mathrm{keV}$.

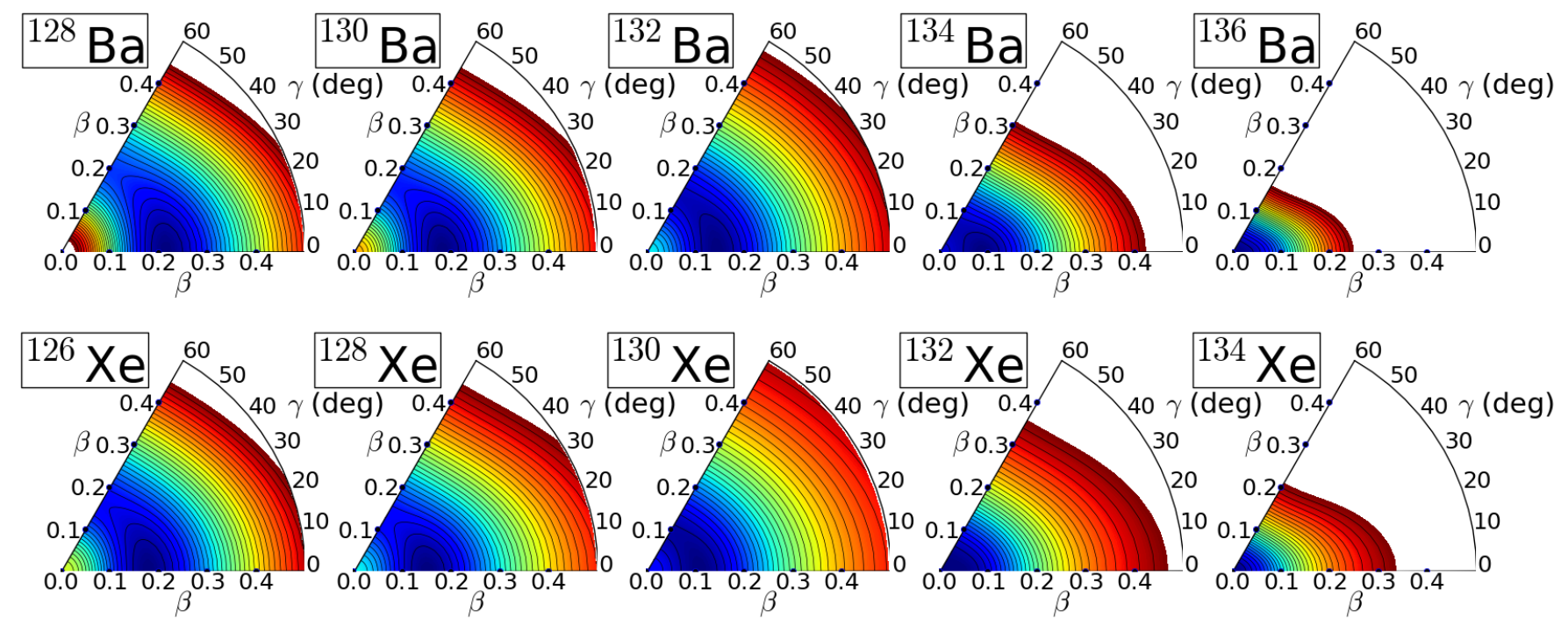

FIG. 6. (Color online) The same as in Fig. 5 , but for the mapped IBM energy surfaces.

One signature of structural evolution in odd-mass nuclei is given by a change in the ground state spin. For example, the positive-parity levels corresponding to $\mathrm{Ba}$ isotopes in Figs. 8(a) and 8(b), display a ground state spin change from $J^{\pi}=1 / 2^{+}$to $3 / 2^{+}$at $N=79$. Both theoretically and experimentally, the $7 / 2_{1}^{+}$and $9 / 2_{1}^{+}$levels at $N=73$ are significantly lower in energy with respect to the neighboring isotope ${ }^{131} \mathrm{Ba}$. This is the consequence of the fact that the $1 g_{7 / 2}$ single-particle orbital becomes lower and closer in energy to the $3 s_{1 / 2}$ and $2 d_{3 / 2}$ orbitals at $N=73$. The $7 / 2_{1}^{+}$and $9 / 2_{1}^{+}$states obtained in the calculations are almost purely $(96 \%$ and
$97 \%$, respectively) composed of the $1 g_{7 / 2}$ configuration. Furthermore, the negative-parity spectra in Figs. 8(c) and $8(d)$ suggest a change in the ground-state spin from $N=75$ to 77 . Similar results are obtained for Xe isotopes (see, Fig. 9). In the case of ${ }^{129-137} \mathrm{La}$ (Figs. 10(a) and 10 (c)) and ${ }^{127-135} \mathrm{Cs}$ (Figs. 11(a) and 11(c)), a reasonable agreement with the experimental data is also observed. Both the theoretical and experimental systematics of the positive-parity states for La (Figs. 10(a) and 10(b)) and Cs (Figs. 11(a) and 11(b)) nuclei, show many levels with very low excitation energy (below $E_{x} \approx 0.2 \mathrm{MeV}$ ) at $N \approx 76$. This reflects structural changes around this neutron number. Indeed, the Gogny-D1M energy sur- 


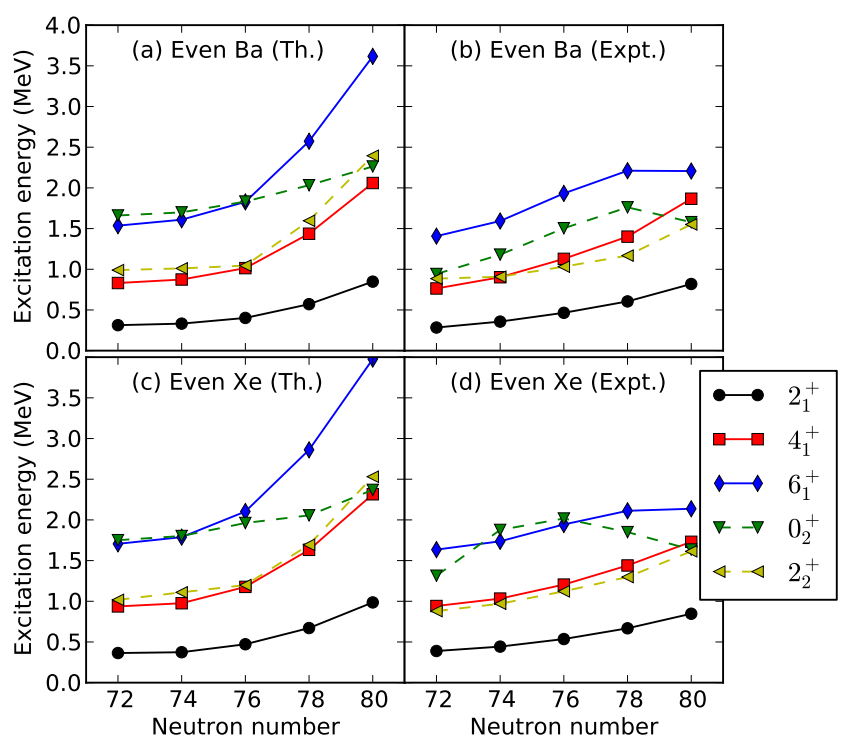

FIG. 7. (Color online) The low-energy excitation spectra obtained for ${ }^{128-136} \mathrm{Ba}$ and ${ }^{126-134} \mathrm{Xe}$ are plotted as functions of the neutron number $N$. Experimental data have been taken from Ref. 48.

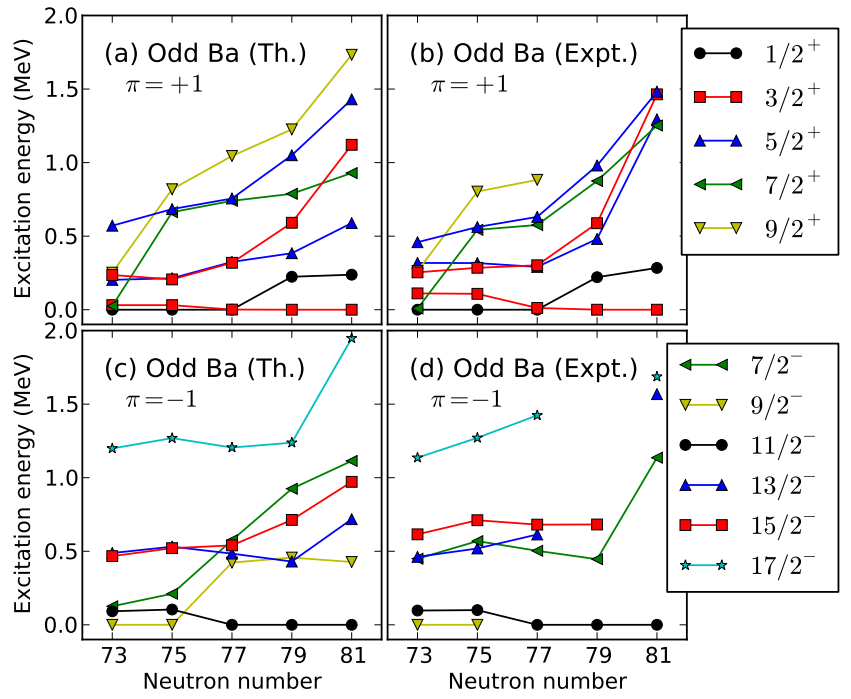

FIG. 8. (Color online) The low-energy positive- $(\pi=+1)$ and negative-parity $(\pi=-1)$ excitation spectra in the odd-mass isotopes ${ }^{129-137} \mathrm{Ba}$ are plotted as functions of the neutron number $N$. Experimental data have been taken from Ref. [48].

faces for the corresponding even-even $\mathrm{Ba}$ and Xe nuclei around $N \approx 76$ suggest that it corresponds to the transition point between nearly spherical and $\gamma$-soft shapes (see, Fig. 5).

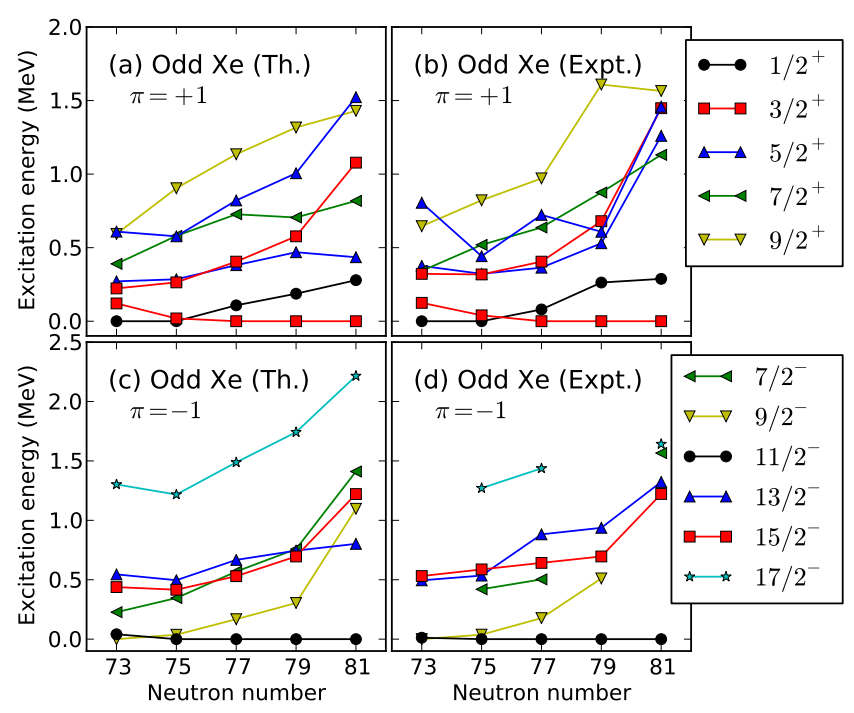

FIG. 9. (Color online) The same as in Fig. 8, but for the odd-mass Xe isotopes.

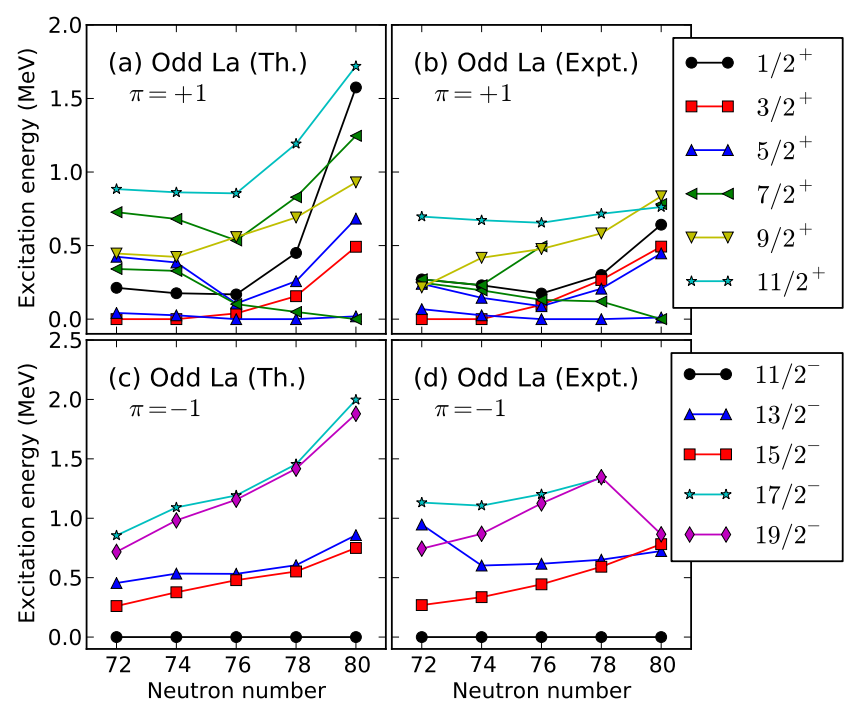

FIG. 10. (Color online) The same as in Fig. 8 but for the odd-mass La isotopes.

\section{B. Systematics of electromagnetic properties}

The $B(E 2)$ transition rates between low-lying positiveparity states where experimental data are available are shown in Fig. 12 For each of the considered isotopic chains, the $B(E 2)$ values increase as the valence nucleon number increases reflecting the development of quadrupole collectivity in the corresponding even-even systems. The computed $B(E 2)$ values appear to be in a reasonable agreement with the experiment. However, the $B\left(E 2 ; 5 / 2_{1}^{+} \rightarrow 1 / 2_{1}^{+}\right)$in odd-mass La nuclei (panel (c) of Fig. 12, for example, decreases (dotted line) while 


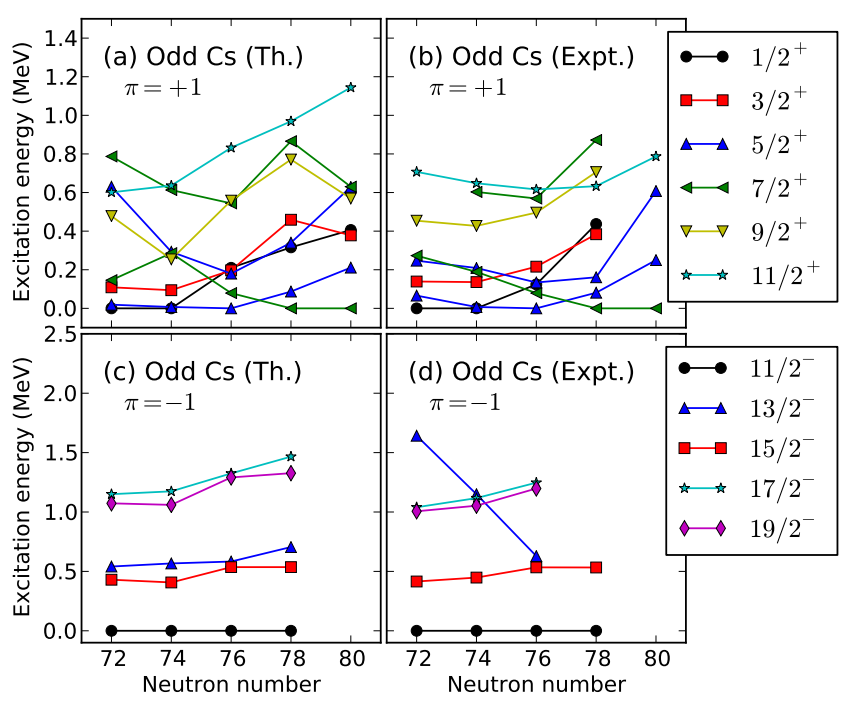

FIG. 11. (Color online) The same as in Fig. 8 but for the odd-mass Cs isotopes.

experimentally (triangle) it increases from $N=74$ to 78 . The analysis of the compositions of the wave functions reveals that the $5 / 2_{1}^{+}$and $1 / 2_{1}^{+}$states are rather similar in structure for the odd- $A$ La isotopes with $N \leq 76$ (both are mainly composed of the $2 d_{5 / 2}$ single-particle configuration) leading to large E2 matrix elements. On the other hand, for La isotopes with $N \geq 78$ the computed wave functions have rather different compositions, i.e., the dominant components of the $5 / 2_{1}^{+}$and $1 / 2_{1}^{+}$states are the $1 g_{7 / 2}$ and $2 d_{5 / 2}$ configurations, respectively.

In Fig. 13 the predicted spectroscopic quadrupole $Q_{J}$ moments corresponding to the $3 / 2_{1}^{+}$and $11 / 2_{1}^{-}$states of the odd-mass Ba (panel (a)) and Xe (panel (b)) nuclei are plotted as functions of the neutron number. The spectroscopic quadrupole moments associated with the $5 / 2_{1}^{+}$and $7 / 2_{1}^{+}$states of the odd-mass La (panel (c)) and Cs (panel (d)) nuclei are also shown in the plot. The predicted $Q_{J}$ values agree reasonably well with the available experimental data 52] exception made of the lighter $(N=75$ and 77$)$ Xe isotopes.

The magnetic moments $\mu_{1 / 2_{1}^{+}}, \mu_{3 / 2_{1}^{+}}$and $\mu_{11 / 2_{1}^{-}}$obtained for the odd- $A$ Ba (panel (a)) and Xe (panel (b)) nuclei are plotted in Fig. 14. The $\mu_{1 / 2_{1}^{+}}, \mu_{5 / 2_{1}^{+}}$and $\mu_{7 / 2_{1}^{+}}$ moments obtained for the odd-mass La (panel (c)) and Cs (panel (d)) nuclei are also included in the plot. The comparison with the available experimental data [52] reveals a reasonable agreement both in magnitude and sign.

\section{Detailed level schemes of selected odd- $A$ nuclei}

In this section, we consider a selected set of odd-mass nuclei with available experimental data. For those nuclei we present more detailed level schemes and electro-
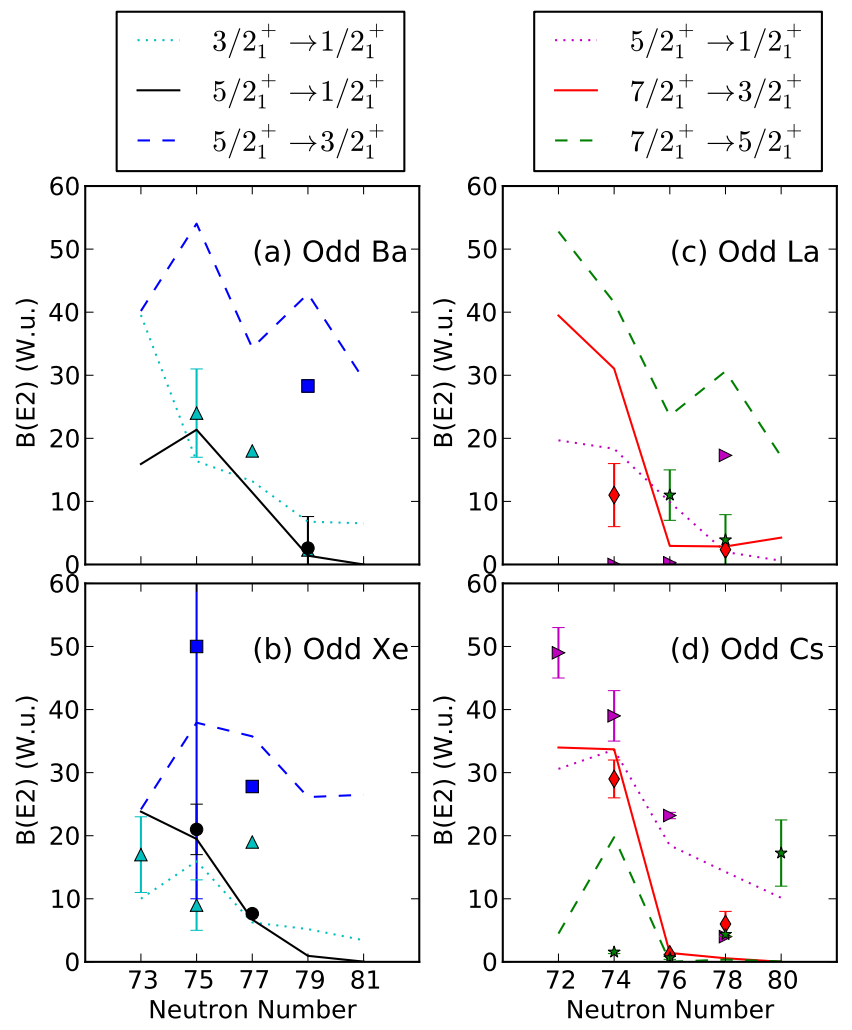

FIG. 12. (Color online) The transition probabilities $B\left(E 2 ; 3 / 2_{1}^{+} \rightarrow 1 / 2_{1}^{+}\right), B\left(E 2 ; 5 / 2_{1}^{+} \rightarrow 1 / 2_{1}^{+}\right)$and $B\left(E 2 ; 5 / 2_{1}^{+} \rightarrow 3 / 2_{1}^{+}\right)$obtained for the odd-mass nuclei ${ }^{129-137} \mathrm{Ba}$ (a) and ${ }^{127-135} \mathrm{Xe}(\mathrm{b})$, and the transition probabilities $B\left(E 2 ; 5 / 2_{1}^{+} \rightarrow 1 / 2_{1}^{+}\right), B\left(E 2 ; 7 / 2_{1}^{+} \rightarrow 3 / 2_{1}^{+}\right)$and $B\left(E 2 ; 7 / 2_{1}^{+} \rightarrow 5 / 2_{1}^{+}\right)$obtained for the odd-mass nuclei ${ }^{129-137} \mathrm{La}$ (c) and ${ }^{127-135} \mathrm{Cs}$ (d) are plotted as functions of the neutron number. The experimental $B\left(E 2 ; 3 / 2_{1}^{+} \rightarrow\right.$ $\left.1 / 2_{1}^{+}\right)$(filled circles), $B\left(E 2 ; 5 / 2_{1}^{+} \rightarrow 1 / 2_{1}^{+}\right)$(squares), $B\left(E 2 ; 5 / 2_{1}^{+} \rightarrow 3 / 2_{1}^{+}\right)$(triangles), $B\left(E 2 ; 7 / 2_{1}^{+} \rightarrow 3 / 2_{1}^{+}\right.$) (diamonds) and $B\left(E 2 ; 7 / 2_{1}^{+} \rightarrow 5 / 2_{1}^{+}\right)$(asterisks) values are taken from Ref. 48.

magnetic transition rates. In particular, we will consider ${ }^{135} \mathrm{Ba},{ }^{129,131} \mathrm{Xe},{ }^{133} \mathrm{La}$ and ${ }^{131,133} \mathrm{Cs}$ taken, as illustrative examples. We will also compare with the results obtained in Ref. 29. The positive- and negative-parity energy spectra provided by our calculations are depicted in Figs. 15 20, The corresponding $B(E 2)$ and $B(M 1)$ values can be found in Tables IIIVIII

Let us first discuss the odd- $N$ nuclei ${ }^{135} \mathrm{Ba}$ and ${ }^{129,131} \mathrm{Xe}$, where the low-lying states are based mainly on the $3 s_{1 / 2}, 2 d_{3 / 2}$ (for positive-parity) and $1 h_{11 / 2}$ (for negative-parity) single-particle configurations. As can be seen from Fig. 15 , exception made of the $3 / 2_{3}^{+}$and $5 / 2_{3}^{+}$ non-yrast states, the positive- and negative-parity energy spectra obtained for ${ }^{135} \mathrm{Ba}$ compare well with the experimental ones. Similar results have been obtained in Ref. [29]. Furthermore, the results obtained in this study 

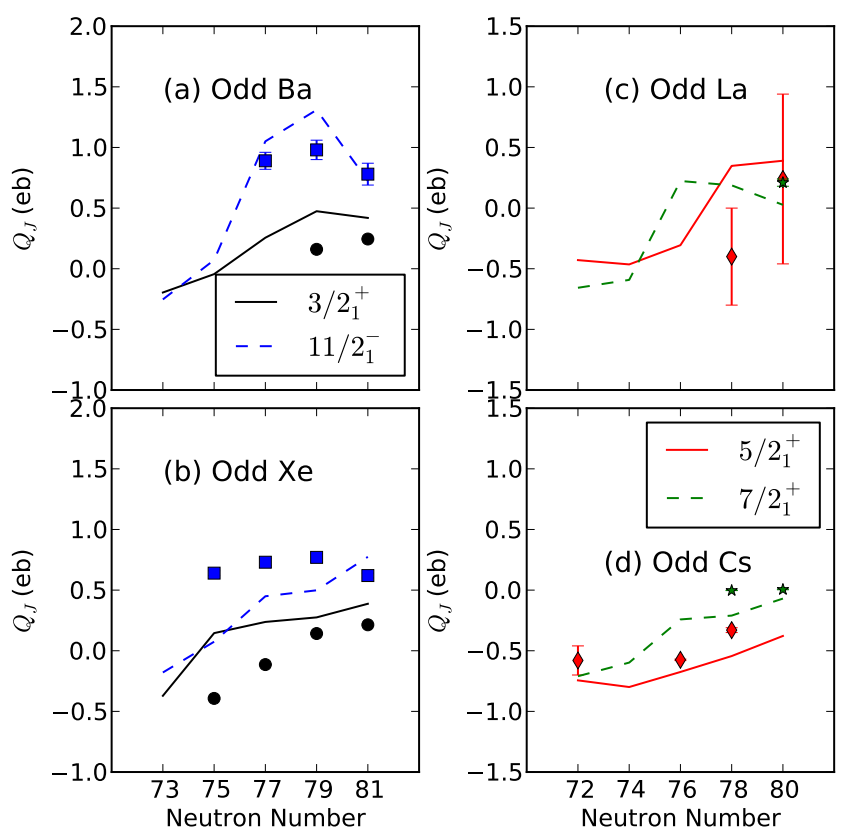

FIG. 13. (Color online) The spectroscopic quadrupole moments $Q_{3 / 2_{1}^{+}}$and $Q_{11 / 2_{1}^{-}}$obtained for ${ }^{129-137} \mathrm{Ba}$ (a) and ${ }^{127-135} \mathrm{Xe}(\mathrm{b})$ and the spectroscopic quadrupole moments $Q_{5 / 2_{1}^{+}}$and $Q_{7 / 2_{1}^{+}}$obtained for ${ }^{129-137} \mathrm{La}$ (c) and ${ }^{127-135} \mathrm{Cs}$ (d) are plotted as functions of the neutron number. The experimental $Q_{3 / 2_{1}^{+}}$(filled circles), $Q_{11 / 2_{1}^{-}}$(squares), $Q_{5 / 2_{1}^{+}}$(diamonds) and $Q_{7 / 2_{1}^{+}}$(asterisks) values are taken from Ref. [52].

TABLE III. Comparison between the theoretical and experimental $B(E 2)$ and $B(M 1)$ values in ${ }^{135} \mathrm{Ba}$. Experimental data are taken from Ref. [48.

\begin{tabular}{lcccc}
\hline \hline & \multicolumn{2}{c}{$B(E 2)$ (W.u.) } & \multicolumn{2}{c}{$B(M 1)$ (W.u.) } \\
\cline { 2 - 5 } & Th. & Expt. & Th. & Expt. \\
\hline $1 / 2_{1}^{+} \rightarrow 3 / 2_{1}^{+}$ & 14 & $4.6(2)$ & 0.0010 & $0.0025(11)$ \\
$1 / 2_{2}^{+} \rightarrow 3 / 2_{1}^{+}$ & 7.2 & $11.7(10)$ & & \\
$3 / 2_{2}^{+} \rightarrow 3 / 2_{1}^{+}$ & 7.5 & $18.0(10)$ & & \\
$3 / 2_{3}^{+} \rightarrow 3 / 2_{1}^{+}$ & 1.1 & $7.0(10)$ & & \\
$5 / 2_{1}^{+} \rightarrow 1 / 2_{1}^{+}$ & 1.4 & $2.6(5)$ & & \\
$5 / 2_{1}^{+} \rightarrow 3 / 2_{1}^{+}$ & 43 & $28.3(10)$ & $3.7 \times 10^{-5}$ & $0.0042(20)$ \\
$7 / 2_{1}^{+} \rightarrow 3 / 2_{1}^{+}$ & 29 & $19.9(8)$ & & \\
$7 / 2_{1}^{+} \rightarrow 5 / 2_{1}^{+}$ & 25 & $12.8(12)$ & 0.0015 & $0.0032(3)$ \\
\hline \hline
\end{tabular}

for the $B(E 2)$ and $B(M 1)$ transition probabilities are in a slightly better agreement with the experimental data than those in Ref. 29].

For both ${ }^{129} \mathrm{Xe}$ (Fig. 16) and ${ }^{131} \mathrm{Xe}$ (Fig. 17), we have obtained a good overall agreement with the experiment. However, the predicted positive-parity levels are more stretched than the experimental ones. Similar results have been found for ${ }^{129} \mathrm{Xe}$ in Ref. [29]. From Tables IV and V] one realizes, that the predicted

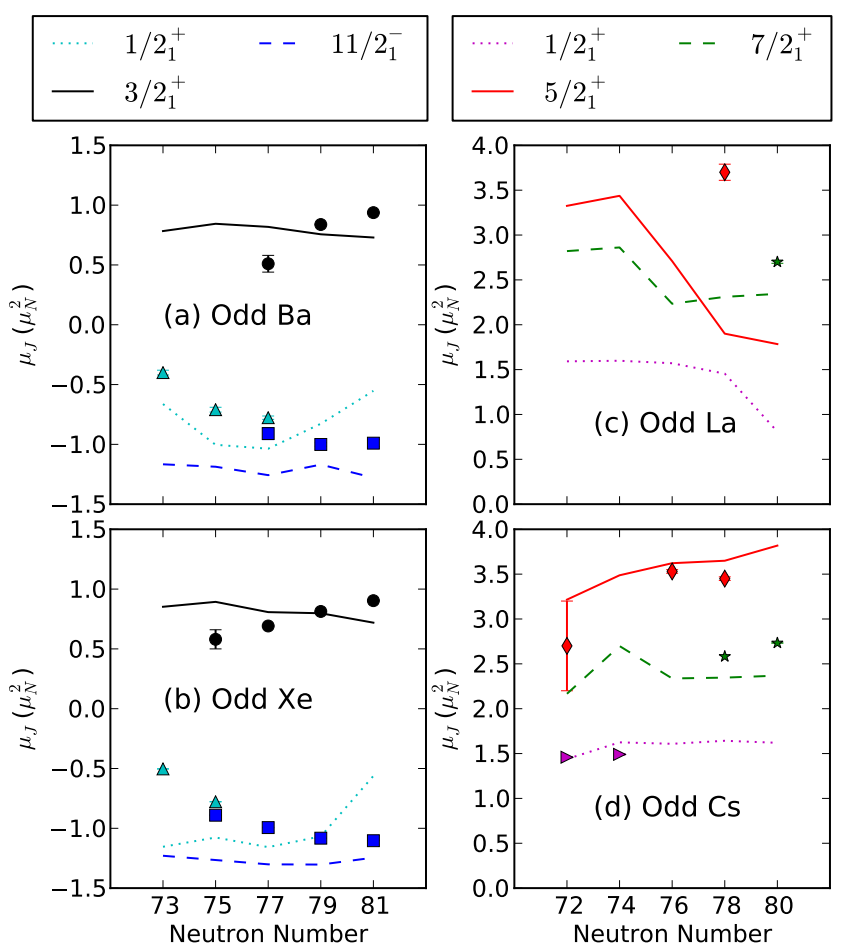

FIG. 14. (Color online) The magnetic moments $\mu_{1 / 2_{1}^{+}}, \mu_{3 / 2_{1}^{+}}$ and $\mu_{11 / 2_{1}^{-}}$obtained for ${ }^{129-137} \mathrm{Ba}$ (a) and ${ }^{127-135} \mathrm{Xe}(\mathrm{b})$ and the magnetic moments $\mu_{1 / 2_{1}^{+}}, \mu_{5 / 2_{1}^{+}}$and $\mu_{7 / 2_{1}^{+}}$obtained for ${ }^{129-137} \mathrm{La}$ (c) and ${ }^{127-135} \mathrm{Cs}$ (d) are plotted as functions of the neutron number. The experimental $\mu_{1 / 2_{1}^{+}}$(filled triangles), $\mu_{3 / 2_{1}^{+}}$(circles), $\mu_{11 / 2_{1}^{-}}$(squares), $\mu_{5 / 2_{1}^{+}}$(diamonds) and $\mu_{7 / 2_{1}^{+}}$ (asterisks) are taken from Ref. [52].

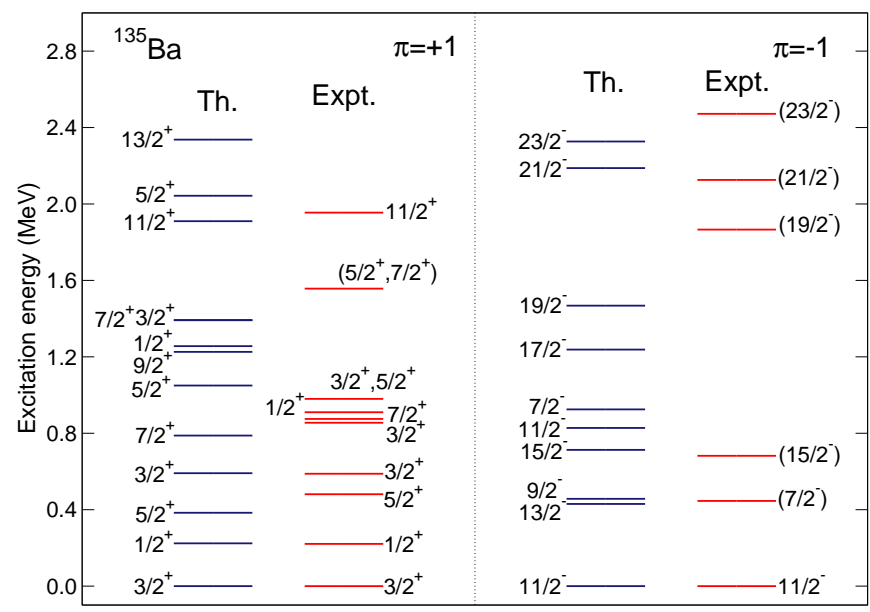

FIG. 15. (Color online) Detailed level scheme for the lowenergy positive- and negative-parity states in ${ }^{135}$ Ba. Experimental data are from Ref. 48].

$B(E 2)$ and $B(M 1)$ transition rates compare well with the experimental ones. However, major discrepancies 


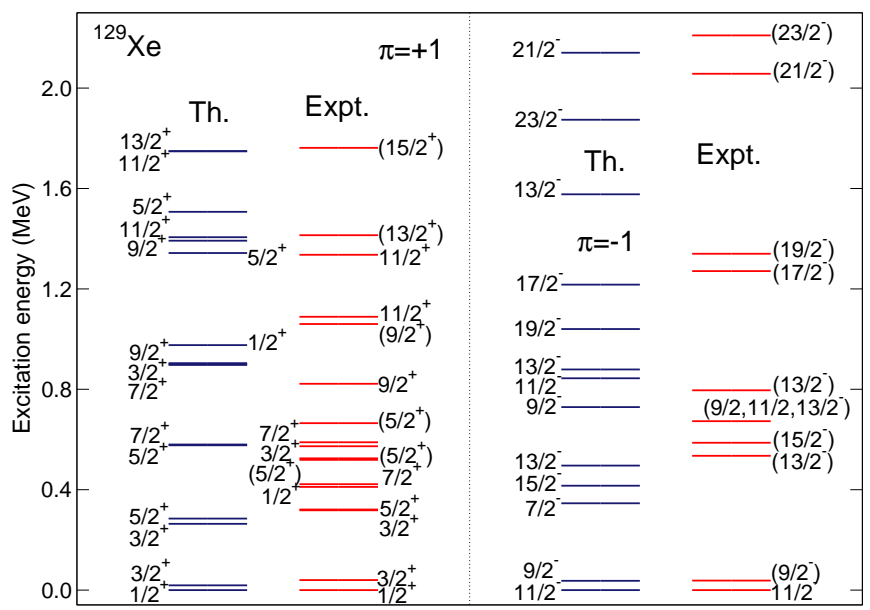

FIG. 16. (Color online) The same as in Fig. 15 but for ${ }^{129}$ Xe.

TABLE IV. The same as in Table III but for ${ }^{129} \mathrm{Xe}$.

\begin{tabular}{lcccc}
\hline \hline & \multicolumn{2}{c}{$B(E 2)($ W.u. $)$} & \multicolumn{2}{c}{$B(M 1)$ (W.u.) } \\
\cline { 2 - 5 } & Th. & Expt. & Th. & Expt. \\
\hline $1 / 2_{2}^{+} \rightarrow 1 / 2_{1}^{+}$ & & & 0.011 & $0.0016(5)$ \\
$1 / 2_{2}^{+} \rightarrow 3 / 2_{1}^{+}$ & 14 & $6.7(23)$ & 0.031 & $0.0039(13)$ \\
$1 / 2_{2}^{+} \rightarrow 3 / 2_{2}^{+}$ & & & 0.011 & $0.0015(5)$ \\
$1 / 2_{2}^{+} \rightarrow 5 / 2_{1}^{+}$ & 1.6 & $1.4(6)$ & & \\
$3 / 2_{1}^{+} \rightarrow 1 / 2_{1}^{+}$ & 16 & $9(4)$ & 0.035 & $0.0281(7)$ \\
$3 / 2_{2}^{+} \rightarrow 1 / 2_{1}^{+}$ & 25 & $23_{-23}^{+25}$ & & \\
$3 / 2_{2}^{+} \rightarrow 3 / 2_{1}^{+}$ & 36 & $17_{-17}^{+27}$ & 0.0078 & $0.003_{-3}^{+4}$ \\
$3 / 2_{3}^{+} \rightarrow 1 / 2_{1}^{+}$ & 7.8 & $>0.2$ & 0.017 & $>0.0001$ \\
$3 / 2_{3}^{+} \rightarrow 1 / 2_{2}^{+}$ & 2.2 & $>5.9$ & 0.0048 & $>0.0026$ \\
$3 / 2_{3}^{+} \rightarrow 3 / 2_{1}^{+}$ & 9.7 & $>1.6$ & 0.0083 & $>0.00071$ \\
$3 / 2_{3}^{+} \rightarrow 3 / 2_{2}^{+}$ & 0.80 & $>3.4$ & 0.015 & $>0.00037$ \\
$3 / 2_{3}^{+} \rightarrow 5 / 2_{1}^{+}$ & 3.8 & $>4.6$ & 0.0083 & $>0.0005$ \\
$5 / 2_{1}^{+} \rightarrow 1 / 2_{1}^{+}$ & 19 & $21(4)$ & & \\
$5 / 2_{1}^{+} \rightarrow 3 / 2_{1}^{+}$ & 38 & $5 \times 10^{1}(4)$ & 0.027 & $0.011(5)$ \\
$5 / 2_{4}^{+} \rightarrow 1 / 2_{1}^{+}$ & 0.00014 & $15.4(19)$ & & \\
\hline \hline
\end{tabular}

are observed for $B\left(E 2 ; 5 / 2_{4}^{+} \rightarrow 1 / 2_{1}^{+}\right)$in ${ }^{129} \mathrm{Xe}$ and $B\left(E 2 ; 7 / 2_{1}^{-} \rightarrow 11 / 2_{1}^{-}\right)$in ${ }^{131} \mathrm{Xe}$. The disagreement can be traced back to the structure of the IBFM wave functions. The $1 / 2_{1}^{+}$and $5 / 2_{4}^{+}$states in ${ }^{129} \mathrm{Xe}$ are dominated by the $3 s_{1 / 2}$ and $2 d_{3 / 2}$ configurations, respectively, leading to rather weak E2 transitions. On the other hand, the negative-parity states are accounted for only by the $1 h_{11 / 2}$ configuration. Therefore, the rather small experimental $B\left(E 2 ; 7 / 2_{1}^{-} \rightarrow 11 / 2_{1}^{-}\right)$transition probability in ${ }^{131} \mathrm{Xe}$ might suggest that some additional contributions, from outside of the employed IBFM model space, should still be taken into account. Indeed, even purely phenomenological IBFM calculations 37] do not reproduce the $B\left(E 2 ; 7 / 2_{1}^{-} \rightarrow 11 / 2_{1}^{-}\right)$transition rate in ${ }^{131} \mathrm{Xe}$. Note, that the $B(E 2)$ and $B(M 1)$ transition rates for ${ }^{129} \mathrm{Xe}$ are similar to the ones obtained in Ref. [29].

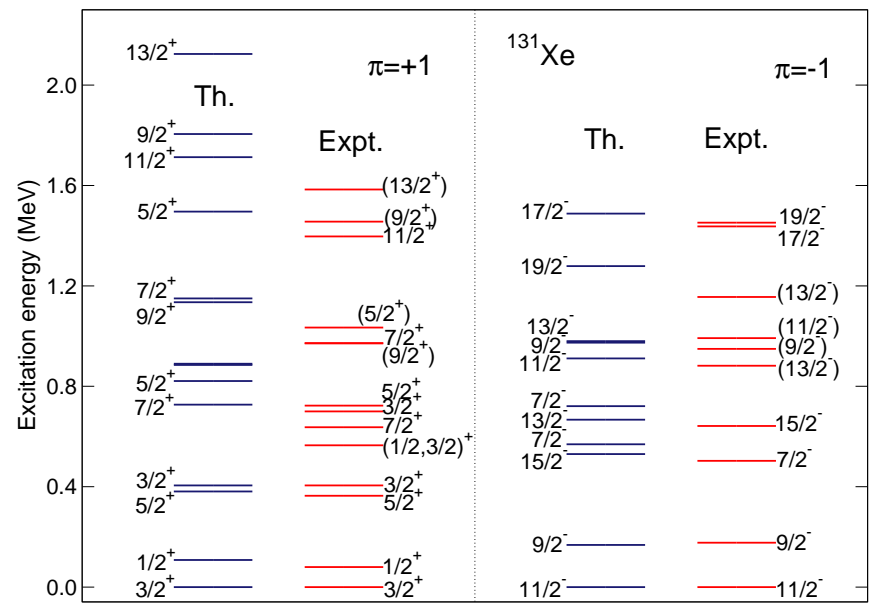

FIG. 17. (Color online) The same as in Fig. 15 , but for ${ }^{131} \mathrm{Xe}$.

TABLE V. The same as in Table III but for ${ }^{131} \mathrm{Xe}$.

\begin{tabular}{lcccc}
\hline \hline & \multicolumn{2}{c}{$B(E 2)($ W.u. $)$} & \multicolumn{2}{c}{$B(M 1)($ W.u. $)$} \\
\cline { 2 - 5 } & Th. & Expt. & Th. & Expt. \\
\hline $1 / 2_{1}^{+} \rightarrow 3 / 2_{1}^{+}$ & 13 & $<37$ & 0.00021 & $>0.032$ \\
$5 / 2_{1}^{+} \rightarrow 1 / 2_{1}^{+}$ & 6.7 & $7.64(24)$ & & \\
$5 / 2_{1}^{+} \rightarrow 3 / 2_{1}^{+}$ & 36 & $27.8(9)$ & 0.0013 & $0.00030(3)$ \\
$3 / 2_{2}^{+} \rightarrow 1 / 2_{1}^{+}$ & 18 & $24_{-24}^{+26}$ & 0.0015 & $0.006(5)$ \\
$3 / 2_{2}^{+} \rightarrow 1 / 2_{1}^{+}$ & 9.8 & $3 . \times 10^{1}(3)$ & 0.00019 & $0.006(6)$ \\
$3 / 2_{3}^{+} \rightarrow 3 / 2_{1}^{+}$ & 7.2 & $10(6)$ & 0.95 & $0.014(4)$ \\
$1 / 2_{2}^{+} \rightarrow 3 / 2_{3}^{+}$ & 1.5 & $10(6)$ & 3.1 & $0.014(4)$ \\
$7 / 2_{1}^{+} \rightarrow 3 / 2_{2}^{+}$ & 0.79 & $1.52(25)$ & & \\
$7 / 2_{1}^{+} \rightarrow 5 / 2_{1}^{+}$ & 6.7 & $1.6(13)$ & 0.0041 & $0.00124(18)$ \\
$7 / 2_{1}^{+} \rightarrow 3 / 2_{1}^{+}$ & 35 & $22.2(19)$ & & \\
$5 / 2_{2}^{+} \rightarrow 3 / 2_{2}^{+}$ & 5.7 & $4_{-4}^{+6}$ & 0.14 & $0.047(5)$ \\
$5 / 2_{2}^{+} \rightarrow 1 / 2_{1}^{+}$ & 30 & $25.7(25)$ & & \\
$5 / 2_{2}^{+} \rightarrow 3 / 2_{1}^{+}$ & 5.5 & $4.8(5)$ & 0.035 & $0.090(9)$ \\
$7 / 2_{2}^{+} \rightarrow 3 / 2_{1}^{+}$ & 0.0018 & $1.6(7)$ & & \\
$9 / 2_{1}^{-} \rightarrow 11 / 2_{1}^{-}$ & 42 & $39(10)$ & 0.094 & $0.00010(4)$ \\
$7 / 2_{1}^{-} \rightarrow 9 / 2_{1}^{-}$ & 0.050 & $0.17(6)$ & 0.00010 & $0.000511(9)$ \\
$7 / 2_{1}^{-} \rightarrow 11 / 2_{1}^{-}$ & 35 & $0.494532(20)$ & & \\
\hline \hline
\end{tabular}

TABLE VI. The same as in Table III but for ${ }^{133} \mathrm{La}$.

\begin{tabular}{lcccc}
\hline \hline & \multicolumn{2}{c}{$B(E 2)($ W.u. $)$} & \multicolumn{2}{c}{$B(M 1)($ W.u. $)$} \\
\cline { 2 - 5 } & Th. & Expt. & Th. & Expt. \\
\hline $1 / 2_{1}^{+} \rightarrow 3 / 2_{1}^{+}$ & 3.4 & $6(3)$ & 0.68 & $0.017(6)$ \\
$1 / 2_{1}^{+} \rightarrow 5 / 2_{1}^{+}$ & 30 & $0.8(3)$ & & \\
$3 / 2_{1}^{+} \rightarrow 5 / 2_{1}^{+}$ & 32 & $>35$ & 0.083 & $>0.026$ \\
$5 / 2_{2}^{+} \rightarrow 5 / 2_{1}^{+}$ & 12 & $2.1(10)$ & 0.14 & $0.0097(8)$ \\
$7 / 2_{1}^{+} \rightarrow 5 / 2_{1}^{+}$ & 24 & $11(4)$ & 0.00013 & $0.0052(9)$ \\
$7 / 2_{1}^{+} \rightarrow 5 / 2_{2}^{+}$ & 21 & $6.1(20)$ & $5.9 \times 10^{-5}$ & $0.00068(16)$ \\
\hline \hline
\end{tabular}

Let us now discuss the odd- $Z$ nuclei ${ }^{133} \mathrm{La}$ and ${ }^{131,133}$ Cs. Their low-energy structures are mainly de- 


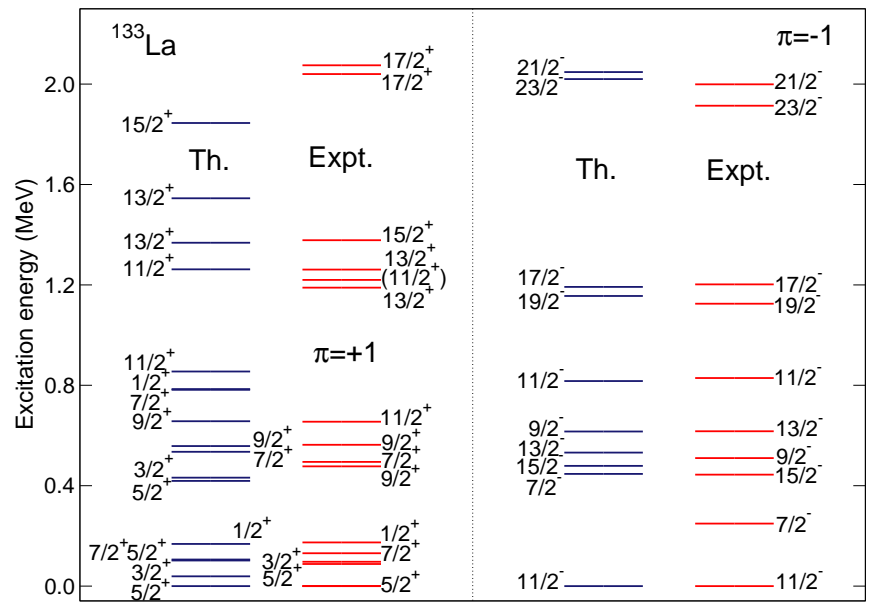

FIG. 18. (Color online) The same as in Fig. 15 but for ${ }^{133}$ La.

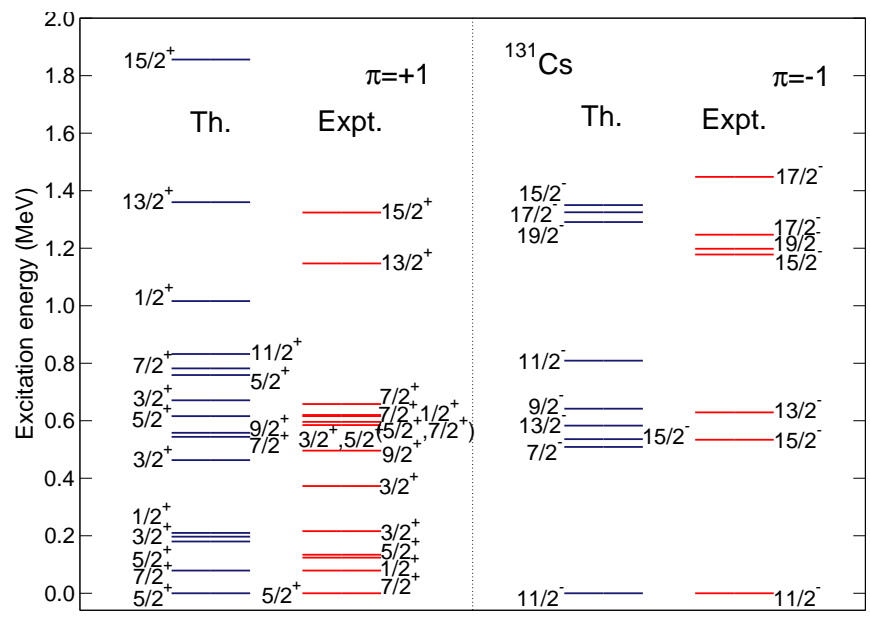

FIG. 19. (Color online) The same as in Fig. 15. but for ${ }^{131}$ Cs.

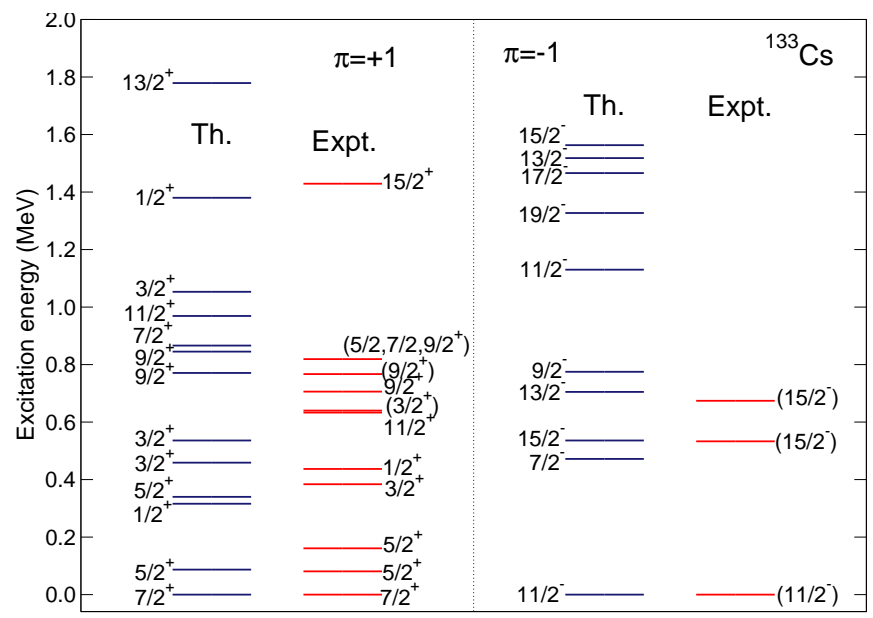

FIG. 20. (Color online) The same as in Fig. 15 but for ${ }^{133}$ Cs.
TABLE VII. The same as in Table III but for ${ }^{131}$ Cs.

\begin{tabular}{lcccc}
\hline \hline & \multicolumn{2}{c}{$B(E 2)($ W.u. } & \multicolumn{2}{c}{$B(M 1)$ (W.u.) } \\
\cline { 2 - 5 } & Th. & Expt. & Th. & Expt. \\
\hline $1 / 2_{1}^{+} \rightarrow 5 / 2_{1}^{+}$ & 55 & $69.5(14)$ & & \\
$1 / 2_{2}^{+} \rightarrow 1 / 2_{1}^{+}$ & \multicolumn{5}{c}{0.076} & $0.0010613(4)$ \\
$1 / 2_{2}^{+} \rightarrow 3 / 2_{1}^{+}$ & 6.3 & $0.09(4)$ & 0.067 & $3.4 \times 10^{-5}(10)$ \\
$1 / 2_{2}^{+} \rightarrow 3 / 2_{2}^{+}$ & 23 & $>0.62$ & 0.0045 & $>5.8 \times 10^{-5}$ \\
$1 / 2_{2}^{+} \rightarrow 5 / 2_{1}^{+}$ & 0.29 & $0.028248(4)$ & & \\
$1 / 2_{2}^{+} \rightarrow 5 / 2_{2}^{+}$ & 7.2 & $0.13835(5)$ & & \\
$3 / 2_{1}^{+} \rightarrow 1 / 2_{1}^{+}$ & 4.9 & $9(5)$ & 0.35 & $0.00339(10)$ \\
$3 / 2_{1}^{+} \rightarrow 5 / 2_{1}^{+}$ & 28 & $0.6(6)$ & 0.13 & $0.00922(5)$ \\
$3 / 2_{1}^{+} \rightarrow 5 / 2_{2}^{+}$ & 1.4 & $>3.9$ & 0.023 & $>4.1 \times 10^{-5}$ \\
$3 / 2_{1}^{+} \rightarrow 7 / 2_{1}^{+}$ & 2.8 & $2.36(3)$ & & \\
$3 / 2_{2}^{+} \rightarrow 1 / 2_{1}^{+}$ & 0.66 & $2.4(4)$ & 0.031 & $0.00057(4)$ \\
$3 / 2_{2}^{+} \rightarrow 3 / 2_{1}^{+}$ & 2.6 & $>2.1$ & 0.0057 & $>7.8 \times 10^{-5}$ \\
$3 / 2_{2}^{+} \rightarrow 5 / 2_{1}^{+}$ & 1.5 & $2.4(9)$ & 0.0033 & $0.00064(20)$ \\
$3 / 2_{2}^{+} \rightarrow 5 / 2_{2}^{+}$ & 4.1 & $0.5(4)$ & 0.0089 & $0.00071(4)$ \\
$3 / 2_{2}^{+} \rightarrow 7 / 2_{1}^{+}$ & 33 & $0.2122(3)$ & & \\
$5 / 2_{2}^{+} \rightarrow 5 / 2_{1}^{+}$ & 0.49 & $3.5(3)$ & 0.017 & $0.000369(17)$ \\
$5 / 2_{2}^{+} \rightarrow 7 / 2_{1}^{+}$ & 44 & $>62$ & 0.0026 & $<2.1 \times 10^{-5}$ \\
$7 / 2_{1}^{+} \rightarrow 5 / 2_{1}^{+}$ & 0.0078 & $0.64(24)$ & 0.0013 & $0.00170(5)$ \\
\hline \hline
\end{tabular}

TABLE VIII. The same as in Table III but for ${ }^{133} \mathrm{Cs}$.

\begin{tabular}{lcccc}
\hline \hline & \multicolumn{2}{c}{$B(E 2)($ W.u. } & \multicolumn{2}{c}{$B(M 1)$ (W.u.) } \\
\cline { 2 - 5 } & Th. & Expt. & Th. & Expt. \\
\hline $5 / 2_{1}^{+} \rightarrow 7 / 2_{1}^{+}$ & 0.49 & $5.8(4)$ & 0.00086 & $0.002381(22)$ \\
$5 / 2_{2}^{+} \rightarrow 7 / 2_{1}^{+}$ & 23 & $28.6(18)$ & 0.0022 & $0.081(4)$ \\
$5 / 2_{2}^{+} \rightarrow 5 / 2_{1}^{+}$ & 0.60 & $1.3 \times 10^{2}(3)$ & 0.0024 & $0.000126(8)$ \\
$3 / 2_{1}^{+} \rightarrow 5 / 2_{2}^{+}$ & 0.024 & $0.12(4)$ & 0.020 & $0.00070(18)$ \\
$3 / 2_{1}^{+} \rightarrow 5 / 2_{1}^{+}$ & 17 & $0.04_{-4}^{+7}$ & 0.12 & $0.011(3)$ \\
$3 / 2_{1}^{+} \rightarrow 7 / 2_{1}^{+}$ & 1.1 & $12(3)$ & & \\
$1 / 2_{1}^{+} \rightarrow 3 / 2_{1}^{+}$ & 8.8 & $>18$ & 0.75 & $>0.024$ \\
$1 / 2_{1}^{+} \rightarrow 5 / 2_{2}^{+}$ & 0.40 & $>4.8$ & & \\
$1 / 2_{1}^{+} \rightarrow 5 / 2_{1}^{+}$ & 43 & $>12$ & & \\
$11 / 2_{1}^{+} \rightarrow 7 / 2_{1}^{+}$ & 25 & $26.1(20)$ & & \\
$3 / 2_{2}^{+} \rightarrow 5 / 2_{2}^{+}$ & 2.7 & $3.6(23)$ & 0.0060 & $0.13(4)$ \\
$3 / 2_{2}^{+} \rightarrow 5 / 2_{1}^{+}$ & 2.0 & $1.0 \times 10^{2}(7)$ & 0.0046 & $0.03(3)$ \\
$3 / 2_{2}^{+} \rightarrow 7 / 2_{1}^{+}$ & 26 & $3.4(7)$ & & \\
$9 / 2_{2}^{+} \rightarrow 5 / 2_{2}^{+}$ & 1.8 & $7.0(17)$ & & \\
$7 / 2_{1}^{+} \rightarrow 7 / 2_{3}^{+}$ & 18 & $6.5(18)$ & 0.039 & $0.016(4)$ \\
\hline \hline
\end{tabular}

scribed by the $1 g_{7 / 2}$ and $2 d_{5 / 2}$ (for positive parity) and $1 h_{11 / 2}$ (for negative parity) orbitals weakly coupled to the corresponding IBM states. The positive- and negative-parity spectra of ${ }^{133}$ La are depicted in Fig. 18. The comparison with the experiment reveals that, as in Ref. [29], our calculations overestimate the positiveparity levels above $1 \mathrm{MeV}$ excitation energy. On the other hand, exception made of the $B\left(E 2 ; 1 / 2_{1}^{+} \rightarrow 5 / 2_{1}^{+}\right)$ and $B\left(E 2 ; 7 / 2_{1}^{+} \rightarrow 5 / 2_{2}^{+}\right)$values, the computed $\mathrm{E} 2$ and M1 transitions listed in Table VI agree reasonably well with the experiment. Similar results have been obtained 
in Ref. [29].

Finally, a reasonable agreement between the computed and the experimental spectra is observed in the case of ${ }^{131} \mathrm{Cs}$ (Fig. 19 ) and ${ }^{133} \mathrm{Cs}$ (Fig. 20). As with ${ }^{133} \mathrm{La}$, the positive-parity states with excitation energy above $1 \mathrm{MeV}$ are overestimated. The calculated $B(E 2)$ and $B(M 1)$ transition rates, shown in Table VII (for ${ }^{131} \mathrm{Cs}$ ) and VIII (for ${ }^{133} \mathrm{Cs}$ ), compare reasonably well with the experimental ones. Note, that the predicted E2 and M1 transition rates agree well with the results of Ref. [29].

\section{SUMMARY AND CONCLUDING REMARKS}

In this work, we have studied the spectroscopic properties of the odd-mass $\gamma$-soft nuclei ${ }^{129-137} \mathrm{Ba},{ }^{127-135} \mathrm{Xe}$, ${ }^{129-137} \mathrm{La}$ and ${ }^{127-135} \mathrm{Cs}$ in terms of the IBFM with the Hamiltonian constructed using the microscopic input provided by the Gogny-D1M HFB approximation. The $(\beta, \gamma)$-deformation energy surfaces for the even-even core nuclei, spherical single-particle energies and occupation numbers for the corresponding odd-mass systems have been computed within the SCMF method, and these quantities have been used to build the IBFM Hamiltonian [25]. The coupling constants of the boson-fermion interaction terms have been fitted to experimental spectra. The IBFM Hamiltonian has been then used to compute spectroscopic properties of the considered odd-mass systems.

For the even-even isotopes ${ }^{128-136} \mathrm{Ba}$ and ${ }^{126-134} \mathrm{Xe}$, the $(\beta, \gamma)$-deformation energy surfaces suggest the empirically observed structural evolution from $\gamma$-soft to nearly spherical shapes as functions of the neutron number. Our (mapped) IBM calculations reproduce the experimental low-energy excitation spectra in both even-even $\mathrm{Ba}$ and Xe nuclei reasonably well. Furthermore, the IBFM calculations provide a reasonable agreement with the available experimental data for the low-energy positive- and negative-parity states as well as the electromagnetic transition rates in the case of the odd-mass ${ }^{129-137} \mathrm{Ba}$, ${ }^{127-135} \mathrm{Xe},{ }^{129-137} \mathrm{La}$ and ${ }^{127-135} \mathrm{Cs}$ nuclei. We have also shown that our results are at the same level of accuracy in reproducing experimental data as those reported in Ref. 29] on the same set of the odd-mass nuclei but using the relativistic mean-field approximation. The results obtained in this study for $\gamma$-soft odd-mass nuclei, as well as the previous ones for the axially-deformed odd-mass $\mathrm{Eu}$ and Sm systems [28, further corroborate the validity of the employed method 25] based on the Gogny-EDF.

Even though the application of the method in its current version is limited to only those nuclei where experimental data are available, the results obtained in this work are promising and open up the possibility for exploring odd-mass nuclear systems in other regions of the nuclear chart. Our next step would be to apply the present method to neutron-rich odd-mass nuclei, and a potential target are those with $A \approx 190-200$, including $\mathrm{Pt}$ and Os nuclei. They are expected to display a rich variety of structural phenomena and sufficient experimental data are already available. Indeed, the corresponding even-even nuclei in this mass region exhibit prolate-oblate shape/phase transitions as well as examples of $\gamma$-softness. It would be, therefore, of interest to study how the presence of an odd particle affects this type of shape/phase transition. Work along this line is in progress, and will be reported elsewhere.

\section{ACKNOWLEDGMENTS}

K.N. acknowledges support from the Japan Society for the Promotion of Science. This work has been supported in part by the QuantiXLie Centre of Excellence. The work of LMR was supported by Spanish Ministry of Economy and Competitiveness (MINECO) Grants No. FPA2015-65929-P and FIS2015-63770-P.
[1] A. Bohr and B. M. Mottelsson, Nuclear Structure, Vol. 2 (Benjamin, New York, USA, 1975) p. 45.

[2] E. Caurier, G. Martínez-Pinedo, F. Nowacki, A. Poves, and A. P. Zuker, Rev. Mod. Phys. 77, 427 (2005).

[3] P. Ring and P. Schuck, The nuclear many-body problem (Berlin: Springer-Verlag, 1980).

[4] M. Bender, P.-H. Heenen, and P.-G. Reinhard, Rev. Mod. Phys. 75, 121 (2003)

[5] D. Vretenar, A. Afanasjev, G. Lalazissis, and P. Ring, Phys. Rep. 409, 101 (2005)

[6] T. Nikšić, D. Vretenar, and P. Ring, Prog. Part. Nucl. Phys. 66, 519 (2011).

[7] R. Rodríguez-Guzmán, J. L. Egido, and L. M. Robledo, Nucl. Phys. A 709, 201 (2002).

[8] B. Bally, B. Avez, M. Bender, and P.-H. Heenen, Phys. Rev. Lett. 113, 162501 (2014).

[9] M. Borrajo and J. L. Egido, The European Physical Journal A 52, 277 (2016).
[10] A. Bohr, Mat. Fys. Medd. Dan. Vid. Selsk. 27, 16 (1953).

[11] K. Mizuyama, G. Colò, and E. Vigezzi, Phys. Rev. C 86, 034318 (2012).

[12] D. Tarpanov, J. Toivanen, J. Dobaczewski, and B. G. Carlsson, Phys. Rev. C 89, 014307 (2014).

[13] D. Tarpanov, J. Dobaczewski, J. Toivanen, and B. G. Carlsson, Phys. Rev. Lett. 113, 252501 (2014).

[14] Y. F. Niu, Z. M. Niu, G. Colò, and E. Vigezzi, Phys. Rev. Lett. 114, 142501 (2015).

[15] E. Litvinova, P. Ring, and V. Tselyaev, Phys. Rev. C 75, 064308 (2007).

[16] E. V. Litvinova and A. V. Afanasjev, Phys. Rev. C 84, 014305 (2011).

[17] F. Iachello and O. Scholten, Phys. Rev. Lett. 43, 679 (1979).

[18] O. Scholten, Prog. Part. Nucl. Phys. 14, 189 (1985).

[19] F. Iachello and P. Van Isacker, The interacting bosonfermion model (Cambridge University Press, Cambridge, 
1991).

[20] F. Iachello, A. Leviatan, and D. Petrellis, Phys. Lett. B 705, 379 (2011).

[21] D. Petrellis, A. Leviatan, and F. Iachello, Ann. Phys. (N.Y.) 326, 926 (2011).

[22] G. R. Jansen, M. D. Schuster, A. Signoracci, G. Hagen, and P. Navrátil, Phys. Rev. C 94, 011301 (2016)

[23] G. De Gregorio, F. Knapp, N. Lo Iudice, and P. Vesely, Phys. Rev. C 94, 061301 (2016).

[24] G. De Gregorio, F. Knapp, N. Lo Iudice, and P. Veselý, Phys. Rev. C 95, 034327 (2017).

[25] K. Nomura, T. Nikšić, and D. Vretenar, Phys. Rev. C 93, 054305 (2016).

[26] F. Iachello and A. Arima, The interacting boson model (Cambridge University Press, Cambridge, 1987).

[27] K. Nomura, T. Nikšić, and D. Vretenar, Phys. Rev. C 94, 064310 (2016).

[28] K. Nomura, R. Rodríguez-Guzmán, and L. M. Robledo, Phys. Rev. C 96, 014314 (2017).

[29] K. Nomura, T. Nikšić, and D. Vretenar, Phys. Rev. C 96, 014304 (2017).

[30] T. Nikšić, D. Vretenar, and P. Ring, Phys. Rev. C 78, 034318 (2008)

[31] C. Alonso, J. Arias, R. Bijker, and F. Iachello, Physics Letters B 144, 141 (1984)

[32] J. Arias, C. Alonso, and R. Bijker, Nuclear Physics A 445, 333 (1985).

[33] M. Cunningham, Nuclear Physics A 385, 204 (1982)

[34] M. Cunningham, Nuclear Physics A 385, 221 (1982)

[35] C. E. Alonso, J. M. Arias, and M. Lozano, Journal of Physics G: Nuclear Physics 13, 1269 (1987)

[36] F. Dellagiacoma, Beta decay of odd mass nuclei in the interacting boson-fermion model, $\mathrm{Ph} . \mathrm{D}$. thesis, Yale Uni- versity (1988).

[37] S. Abu-Musleh, H. Abu-Zeid, and O. Scholten, Nuclear Physics A 927, 91 (2014)

[38] T. Otsuka, A. Arima, and F. Iachello, Nucl. Phys. A 309, 1 (1978)

[39] K. Nomura, N. Shimizu, and T. Otsuka, Phys. Rev. Lett. 101, 142501 (2008)

[40] K. Nomura, N. Shimizu, and T. Otsuka, Phys. Rev. C 81, 044307 (2010).

[41] S. Goriely, S. Hilaire, M. Girod, and S. Péru, Phys. Rev. Lett. 102, 242501 (2009)

[42] J. N. Ginocchio and M. W. Kirson, Nucl. Phys. A 350, $31(1980)$

[43] J. F. Berger, M. Girod, and D. Gogny, Nucl. Phys. A 428, 23 (1984)

[44] L. M. Robledo, R. R. Rodríguez-Guzmán, and P. Sarriguren, Phys. Rev. C 78, 034314 (2008).

[45] R. Rodríguez-Guzmán, P. Sarriguren, L. M. Robledo, and J. E. García-Ramos, Phys. Rev. C 81, 024310 (2010)

[46] T. Otsuka and N. Yoshida, (1985), jAERI-M (Japan At. Ener. Res. Inst.) Report No. 85.

[47] O. Scholten and N. Blasi, Nucl. Phys. A 380, 509 (1982).

[48] Brookhaven National Nuclear Data Center, http://www.nndc.bnl.gov.

[49] R. F. Casten and N. V. Zamfir, Phys. Rev. Lett. 85, 3584 (2000).

[50] F. Iachello, Phys. Rev. Lett. 85, 3580 (2000)

[51] P. Van Isacker and J.-Q. Chen, Phys. Rev. C 24, 684 (1981)

[52] N. Stone, At. Data Nucl. Data Tables 90, 75 (2005). 